PALEO

Revue d'archéologie préhistorique

13 | 2001

Varia

\title{
Exploitation de l'Antilope saïga au Magdalénien en Aquitaine
}

Magdalenian Saiga antelope's exploitation in Aquitaine: methods and archaeological applications

\section{Sandrine Costamagno}

\section{OpenEdition}

Journals

Édition électronique

URL : http://journals.openedition.org/paleo/999

DOI : 10.4000/paleo.999

ISSN : 2101-0420

Éditeur

SAMRA

Édition imprimée

Date de publication : 14 décembre 2001

Pagination : 111-128

ISSN : 1145-3370

\section{Référence électronique}

Sandrine Costamagno, «Exploitation de l'Antilope saïga au Magdalénien en Aquitaine », PALEO [En ligne], 13 | 2001, mis en ligne le 26 mai 2010, consulté le 07 juillet 2020. URL : http://

journals.openedition.org/paleo/999; DOI : https://doi.org/10.4000/paleo.999

Ce document a été généré automatiquement le 7 juillet 2020

\section{(c) (†) $\odot$}

PALEO est mis à disposition selon les termes de la licence Creative Commons Attribution - Pas d'Utilisation Commerciale - Pas de Modification 4.0 International. 


\title{
Exploitation de l'Antilope saïga au Magdalénien en Aquitaine
}

\author{
Magdalenian Saiga antelope's exploitation in Aquitaine: methods and \\ archaeological applications
}

Sandrine Costamagno

1 Originaire d'Eurasie, l'Antilope saïga a connu deux phases de migration au cours du Pléistocène. La première phase d'immigration vers l'ouest et le sud de l'Europe s'amorce au cours d'un épisode climatique continental et sec du stade isotopique 6 (Simms 1987). En France, on trouve, durant cette période, des restes d'Antilope saïga à Combe-Grenal (Delpech et Heintz, 1976 ; Delpech et Prat, 1995) et à l'Abri Suard (David, 1952 ; David et Prat, 1965). C'est au cours du stade isotopique 2 avec la mise en place de conditions climatiques extrêmement arigoureuses que la seconde phase d'immigration débute. L'Antilope saïga se dirige alors vers le sud-ouest de la France où elle va trouver refuge jusqu'à l'amorce des premières phases de réchauffement (Delpech, 1989). Bien que des restes d'Antilope saïga aient été signalés dans des niveaux solutréens (Castel 1999 ;Crégut-Bonnoure, 1991), c'est au Magdalénien que l'Antilope saïga abonde. On la retrouve, en effet, dans de nombreuses régions: Massif central (Delpech, 1989), Provence (Crégut-Bonnoure et Gagnière, 1981), Nord de l'Espagne (Altuna et Mariezkurrena, 1996) ou bien encore Aquitaine (Delpech, 1983). Parmi ces régions, cette dernière est tout à fait originale puisque c'est la seule zone où l'Antilope saïga a joué un rôle non négligeable dans l'alimentation des Magdaléniens. Comment cette espèce a-telle influé sur les stratégies de chasse et plus largement sur les modes d'exploitation des territoires des hommes ayant vécu dans cette région? C'est la question à laquelle nous essaierons de répondre en abordant plusieurs points.

2 En premier lieu, le succès d'une chasse dépend largement de l'habileté du groupe à prédire les réactions des espèces chassées dans un environnement donné. La connaissance de l'écologie et de l'éthologie de l'Antilope saïga est donc primordiale pour cerner les stratégies de chasse mises en œuvre par ces Magdaléniens. L'étude du mode de recrutement des individus chassés permet de mettre en évidence un choix préférentiel ou non, des hommes, en faveur d'animaux de sexe ou d'âge particulier et 
par là-même de documenter une chasse sélective ou opportuniste du gibier. Dans la seconde partie, une méthode permettant d'estimer l'âge des antilopes saïga d'après les dents est donc présentée ainsi qu'une étude sur le dimorphisme sexuel de cet animal. La troisième partie est consacrée plus particulièrement aux données archéologiques. Bien que tenant compte des différents gisements aquitains ayant livré des restes d'Antilope saïga, la discussion est axée principalement sur deux sites : Moulin-Neuf et Saint-Germain-la-Rivière qui sont les deux seuls à avoir fait l'objet d'études archéozoologiques et taphonomiques détaillées (Costamagno 1999, 2000). L'influence de la chasse à l'Antilope saïga sur les modes d'exploitation des territoires par les hommes est ensuite discutée.

\section{Ethologie de l'Antilope saïga}

\section{Habitat}

3 L'habitat de la Saïga est extrêmement limité. Elle peuple les plaines et évite les régions montagneuses et les terrains plus ou moins accidentés. En revanche, elle n'accorde aucune importance à l'altitude absolue. Son aire de répartition actuelle est cantonnée aux zones steppiques arides et semi-désertiques. Son allure particulière, l'amble, lui permet d'atteindre de grandes vitesses, mais lui rend difficiles les sauts, l'obligeant à contourner tout obstacle rencontré.

\section{Croissance et espérance de vie}

4 Les antilopes saïga ont une espérance de vie qui varie selon les régions occupées. D'après A.G. Bannikov (1967), la forte espérance de vie (9-10 ans) observée sur la rive droite de la Volga serait liée à l'absence de loups. En revanche, dans le Kazakhstan, les mâles ne vivent généralement pas plus de cinq ans, alors que les femelles peuvent atteindre l'âge de onze ans. Cette forte disproportion entre mâles et femelles serait due aux loups attaquant plus facilement les mâles épuisés en hiver.

5 Jusqu'à l'âge de sept mois, les mâles voient leur poids augmenter rapidement. Durant les quatre mois suivants qui correspondent aux mois d'hiver, la croissance est ralentie. Ils atteignent leur taille adulte vers 19 mois.

6 Durant les 11 premiers mois, la croissance des femelles est identique à celle des mâles. Contrairement à ces derniers, durant leur second printemps, elles ne grandissent pas car elles donnent naissance à leur première progéniture. Ce n'est qu'en juillet que leur croissance se poursuit. Tout comme les mâles, elles atteignent leur poids adulte vers le dix-neuvième mois.

\section{Périodes d'accouplement et mise bas}

7 Les indications données dans ce paragraphe proviennent principalement de l'étude faite par A.G. Bannikov et ses collaborateurs $(1963 ; 1967)$ sur des populations actuelles d'antilopes saïga.

8 Actuellement, la période d'accouplement varie d'ouest en est. Sur la rive droite de la Volga, elle s'échelonne de début novembre à mi-décembre, au Kazakhstan, elle est décalée de 10 jours et en Mongolie de 20. La gestation dure cinq mois et la femelle 
donne généralement naissance à un ou deux petits qu'elle allaite jusqu'à la fin du mois d'août. Les femelles se regroupent pour la mise bas qui se situe de fin avril à début mai sur la rive droite de la Volga, mi-mai dans le Kazakhstan et fin mai en Mongolie. Des mâles sont alors présents mais en très faibles proportions (environ 2 à $3 \%$ ). Dans leur localisation, ce sont les regroupements les plus stables.

\section{Régime alimentaire et condition physique}

Le régime alimentaire de l'Antilope saïga varie au cours de l'année et selon la région de peuplement (lichens et graminées). Du printemps à l'automne, les animaux paissent toute la journée et parcourent quotidiennement plusieurs dizaines de $\mathrm{km} 2$ en fonction de la qualité des pâturages et de la dimension des troupeaux. En revanche, en été, c'est la recherche de l'eau qui influe sur la répartition de ces animaux, les saïgas préférant consommer des plantes succulentes que boire de l'eau (Bannikov 1967).

Le poids moyen de l'Antilope saïga est fort variable d'une région à l'autre. Actuellement, les mâles vivant sur les rives de la Volga pèsent en moyenne $43 \mathrm{~kg}$ et les femelles $31 \mathrm{~kg}$ tandis que le poids des populations de Mongolie varie de 26 à $32 \mathrm{~kg}$. La masse corporelle se modifie fortement au cours de l'année. Comme on peut le voir sur la figure 1, la condition physique de la Saïga (mâle ou femelle) est constante durant les mois de juillet et d'août. En automne, quand la température devient plus fraîche et que la végétation croît, le poids des antilopes augmente rapidement.

11 Au début de la saison du rut (fin novembre), les mâles sont dans leur meilleure condition physique (accumulation de graisse dans la partie postérieure). Ensuite, au cours de la période d'accouplement, ils se nourrissent peu et se dépensent beaucoup. La perte de poids engendrée par le rut est donc beaucoup plus rapide que l'augmentation enregistrée durant l'automne. Durant tout l'hiver, les mâles restent maigres et ce n'est qu'à partir de la fin du mois de mars qu'ils commencent à reconstituer leur réserve de graisse.

12 Le profil de la courbe de l'indice de condition physique pour les femelles est, en terme général, comparable à celui des mâles. Cependant, les femelles continuent à gagner du poids pendant la saison du rut, la perte de poids ne commençant qu'au mois de janvier (premier mois de la gestation). Durant le mois d'avril, les femelles sont dans leur plus mauvaise condition; elles commencent à reprendre du poids dès la mi-mai et atteignent leur condition moyenne à la fin du mois de juin (un mois après les mâles).

Ce type de courbe prend en compte le poids de l'animal qui est parfois lié à la quantité de graisse. En effet, comme on le remarque sur la figure 1, les femelles qui, en décembre, sont dans leur meilleure condition physique possèdent un indice comparable à celui des mâles durant la saison du rut (mauvaise condition). Cet indice indique que les mâles sont plus lourds que les femelles. En revanche, en ce qui concerne la graisse emmagasinée, les conclusions sont tout autres puisque les femelles, bien que plus légères que les mâles, sont probablement plus grasses que ces derniers et donc plus recherchées par les chasseurs (Speth, 1983). 
Figure 1 : Condition physique de l'Antilope saïga en fonction des saisons - a- femelles, b- mâles (l'indice de condition physique correspond au rapport entre le poids de l'animal et la longueur du corps) (d'après Bannikov 1967, p. 129).

Figure 1 : Seasonal changes in the condition of Saiga antelope - a-females, $b$ - males (index of condition is defined as the ratio of body weight to body length) (after Bannikov 1967, p. 129).
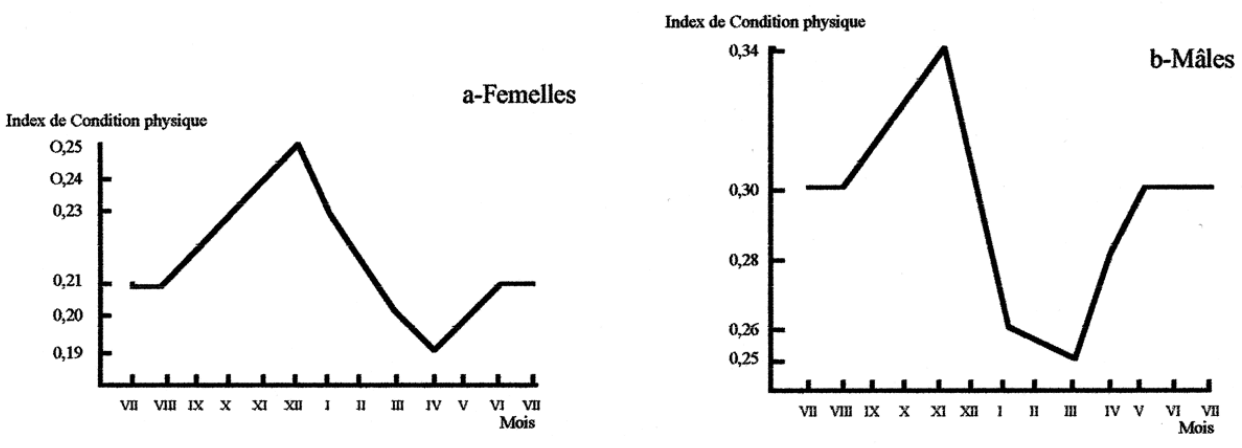

\section{Migrations saisonnières et composition des troupeaux}

Comme dans le paragraphe précédent, nous nous référons pour l'essentiel aux travaux de A.G. Bannikov et ses collaborateurs $(1963$; 1967). L'Antilope saïga est un animal en constant déplacement. Ses migrations saisonnières varient selon son aire de répartition et selon les années. Ainsi dans les steppes européennes, elles sont plus on moins sédentaires et ne se dirigent vers le sud que lors d'hivers vigoureux avec forte chute de neige. En revanche, dans le Kazakhstan, elles effectuent, en automne, une migration vers le sud passant de zones semi-désertiques à des zones désertiques (Pfeffer 1964). Les hivers peu enneigés, elles migrent vers le nord à la recherche de meilleurs pâturages. Ce phénomène n'est pas commun à toutes les populations d'Antilope saïga, il est entièrement régulé par le régime des pluies et l'état des pâturages (Bannikov 1958). Ainsi, sur les rives droites de la Volga, la migration débute habituellement en décembre, mais, en fonction des conditions météorologiques, elle peut se dérouler en novembre ou en janvier. Lors d'hivers peu enneigés, les Saïgas restent sédentaires. Les migrations d'été sont liées à la sécheresse. Les périodes de migration d'été varient donc fortement selon les années. Lors des migrations, il semble que ce soient d'abord de grands troupeaux constitués essentiellement de femelles et de leurs petits qui migrent, suivis par des groupes plus restreints d'animaux mâles. Ainsi, on peut affirmer que "les migrations régulières suivant des itinéraires fixes ne sont pas propres à la Saïga. Cependant, les migrations liées à la recherche des pâturages et des points d'eau font partie intégrante de la biologie de l'espèce." (Bannikov 1958, p 221).

En relation avec les déplacements, la composition et la taille des troupeaux varient fortement au cours de l'année (tabl. 1). 
Tableau 1 : Taille et composition des troupeaux d'Antilope saïga selon les cycles saisonniers. Table 1 : Size and composition of Saiga antelope herds according to seasons.

\begin{tabular}{|l|l|l|l|}
\hline Saison & Taille & Composition & Causes \\
\hline Décembre & $\begin{array}{l}\text { Petit } \\
\text { moyen }\end{array}$ & $\begin{array}{l}\text { Mixte } \\
\text { Mâles }\end{array}$ & $\begin{array}{l}\text { Rut } \\
\text { Jeunes }\end{array}$ \\
\hline $\begin{array}{l}\text { Hiver } \\
\text { (janvier, février) }\end{array}$ & grand & $\begin{array}{l}\text { Mixte } \\
\text { Mâles }\end{array}$ & $\begin{array}{l}\text { Chute de neige } \\
\text { Mauvaise condition physique }\end{array}$ \\
\hline $\begin{array}{l}\text { Début printemps } \\
\text { (mars, début avril) }\end{array}$ & Moyen à grand & Mixte & Migration \\
\hline mi-avril & Moyen à grand & $\begin{array}{l}\text { Femelles } \\
\text { Mâles }\end{array}$ & Mise bas \\
\hline $\begin{array}{l}\text { Fin printemps } \\
\text { (mai, juin) }\end{array}$ & Grand à petit & $\begin{array}{l}\text { Mixte } \\
\text { Femelles }\end{array}$ & Mobilité ralentie \\
\hline $\begin{array}{l}\text { Eté } \\
\text { (juillet, août) }\end{array}$ & Petit généralement & Mixte & Fonction de la qualité des pâturages \\
\hline $\begin{array}{l}\text { Automne } \\
\text { (septembre à novembre) }\end{array}$ & Moyen à grand & Mixte & Fonction des précipitations \\
\hline
\end{tabular}

Hiver (décembre et janvier) : le mois de décembre est caractérisé par la formation de petits troupeaux (1 à 20 têtes) à mettre en relation avec la période de rut. Chaque mâle adulte constitue des "harems" de 10 à 15 femelles tandis que les mâles nés l'année précédente peuvent se regrouper en hardes plus ou moins importantes. Durant le mois de janvier, la taille des troupeaux augmente progressivement. Certains auteurs signalent la présence de hardes mixtes au cours de l'hiver (Vasenko 1950 cité dans Bannikov 1967), alors que d'autres observent des troupeaux constitués uniquement de mâles (Sokolov, 1951 cité dans Bannikov 1967). Après les chutes de neige, des concentrations intenses sont généralement observées.

Début du printemps (fin mars/début avril) : c'est au cours de cette période que les migrations ont lieu. La taille des troupeaux est variable. Ceux de taille moyenne sont les plus souvent rencontrés (50 à 100 têtes). Toutes les hardes sont mixtes; toutes les classes d'âge sont présentes. La seconde moitié du mois d'avril est caractérisée par une migration à grande échelle vers la zone de mise bas. Les trois tailles de hardes sont rencontrées dans des proportions équivalentes. Des concentrations de plus de 10000 animaux peuvent être observées.

Période de mise bas : les femelles se regroupent. Ces hardes ne comportent que 2 à $4 \%$ de mâles. Elles continuent à se déplacer jusqu'au dernier jour, ce qui crée mécaniquement des concentrations énormes. Cependant, la taille des agrégats varie selon le degré de sécheresse ; lors d'années sèches, la dispersion est plus grande. Après la mise bas, les femelles avec leurs petits restent groupées. Au moindre danger, elles vont se disperser dans des directions différentes, alors que durant les autres périodes de l'année, elles ont tendance à s'enfuir toutes dans la même direction.

Fin du printemps : dès la mi-mai, les femelles accompagnées de leurs nouveau-nés quittent la zone de mise bas et forment de grandes concentrations. Durant la seconde moitié du mois de mai, la taille des groupes diminue ( 1 à 19 têtes). Les mâles sont généralement avec les femelles les plus fortes mais parfois, des groupes constitués uniquement de mâles peuvent se créer. La mobilité des femelles ralentie par la présence des jeunes recommence à augmenter durant le mois de juin qui est également caractérisé par des hardes mixtes.

19 Été (juillet et août): les petites hardes prévalent suivies par des hardes de taille moyenne. La dispersion est fonction de la qualité des pâturages qui diminue progressivement au cours de l'été et qui peut varier selon les années mais également 
selon les régions occupées. La plupart des hardes sont mixtes. Dès le mois d'août, les jeunes se séparent de leur mère et constituent de petits groupes. Cette désagrégation correspond à la fin de la période de lactation.

Automne (septembre à novembre): les hardes sont plus grandes (taille moyenne principalement et grande) qu'en été. Les concentrations sont en relation avec l'abondance de la végétation succulente qui est elle-même fonction du degré de pluviométrie enregistrée durant ces mois-là.

21 En résumé, l'Antilope saïga est un Ongulé grégaire. La taille des hardes est, cependant, fortement variable selon les périodes de l'année. En décembre (période de rut) et durant les mois d'été, les petits groupes dominent (1 à 19 individus). Les grands et très grands troupeaux (201 à plus de 1000 individus) sont caractéristiques des mois d'hiver et des migrations de printemps et d'automne. La fréquence des hardes de taille moyenne (20 à 200 individus) ne varie pas saisonnièrement : c'est le mode typique de vie de cette espèce.

\section{Méthode d'estimation d'âge des restes dentaires et dimorphisme sexuel de l'Antilope saïga}

\section{Méthode d'estimation d'âge}

Les profils de mortalité sont particulièrement utiles pour la compréhension des stratégies d'approvisionnement des ressources carnées des Hommes préhistoriques (e.g. Alvard et Kaplan 1991; Blumenschine 1991; Fischer 1987; Frison 1991; Koike et Ohtaishi 1985, 1987; Lyman 1991; Speth 1991; Stiner 1991b). En archéologie, les méthodes d'estimation d'âge s'appuient essentiellement sur les dents, seul élément squelettique à évoluer assez régulièrement tout au long de la vie de l'animal. La méthode d'estimation d'âge de l'Antilope saïga présentée ici est basée sur les séquences d'éruption et l'usure des dents inférieures. Cette méthode a été élaborée en comparant les observations effectuées par A. G. Bannikov (1967) sur des antilopes actuelles à des séries dentaires et dents isolées archéologiques provenant du site de Saint-Germain-laRivière.

Dès leur naissance, les nouveau-nés portent les dents déciduales. À la fin du second mois, elles s'arrêtent de croître; la $\mathrm{M}_{1}$ est au deux tiers de sa hauteur adulte. Durant le troisième mois, cette dernière atteint sa hauteur définitive et la $\mathrm{M}_{2}$ commence à sortir. Sa croissance stoppe durant le septième mois, alors que la $\mathrm{M}_{3}$ commence à apparaitre. Au neuvième mois, la formule dentaire est identique, l'usure étant plus intense. Vers le treizième/quatorzième mois, les dents déciduales paraissent extrêmement usées : elles tombent au cours du quinzième mois sur un laps de temps très court (une semaine environ). La $\mathrm{D}_{4}$ est la première remplacée, suivie par les deux autres. Au dixseptième mois, la croissance des prémolaires est déjà presque complète. Elle s'achève comme celle de la $\mathrm{M}_{3}$ durant le dix-neuvième mois. Entre dix-huit mois et deux ans, les individus sont très difficiles à différencier : on observe, parfois, à la fin de la seconde année, la disparition de la fossette de l'hypoconulide de la $\mathrm{M}_{3}$, le lobe mésial de la $\mathrm{M}_{1}$ étant totalement abrasé (disparition de la fossette). Durant la troisième année, la surface occlusale de la $M_{1}$ est complètement usée (disparition de la fossette postérieure); la $\mathrm{M}_{2}$ est caractérisée par l'absence de fossette au niveau du lobe 
antérieur. Jusqu'à six ans, l'usure des surfaces occlusales reste inchangée. Entre sept et huit ans, la $M_{2}$ ne présente plus ni crête, ni fossette et la $M_{3}$ ne possède plus que celle du lobe médian. À partir de neuf ans, l'ensemble des dents est extrêmement usé.

Sur la base de ces observations, nous avons attribué un âge aux séries dentaires de Saint-Germain-la-Rivière. Pour chaque série, l'usure des molaires mais également celle de la $\mathrm{D}_{4}$ et de la $\mathrm{P}_{4}$ ont été examinées et notées selon la codification définie par $\mathrm{S}$. Payne (1973) sur des dents d'Ovicaprinés et appliquée depuis à d'autres espèces comme le Cerf (Lowe 1967) ou la Chèvre Angora (Deniz et Payne 1982). En combinant ces deux observations, il est possible, à partir des stades d'usure, d'estimer l'âge d'une dent isolée. L'application de cette méthode à plusieurs types de dents permet de vérifier la cohérence des résultats et, par conséquent, de tester la fiabilité de la méthode utilisée (Costamagno 1999).

Sur la base de critères précis d'usure, il est possible de distinguer sept classes d'âge :

- Classe I (0-2 mois) : $\mathrm{M}_{1}$ en cours d'éruption

- Classe II (3-6 mois) : $\mathrm{M}_{1}$ atteint la hauteur de la quatrième déciduale, $\mathrm{M}_{2}$ en cours d'éruption

- Classe III (7-16 mois) : croissance complète de la $\mathrm{M}_{2}, \mathrm{M}_{3}$ en cours d'éruption, chute des déciduales. Au niveau des dents jugales isolées, il est impossible de différencier les individus entre 7 et 14 mois des individus entre 15 et 16 . Seul l'examen de la $\mathrm{D}_{4} / \mathrm{P}_{4}$ permet une telle différenciation : IIIA et IIIB.

- Classe IV (17 mois-2 ans) : fossette du lobe antérieur de la $\mathrm{M}_{1}$ absente, parfois fossette du troisième lobe de la $\mathrm{M}_{3}$

- Classe V (3-6 ans) : les deux fossettes de la $\mathrm{M}_{1}$ absentes, disparition de la fossette du lobe antérieur de la $\mathrm{M}_{2}$

- Classe VI (7-8 ans) : $M_{1}$ et $M_{2}$ totalement usées, seule la fossette médiane de la $M_{3}$ subsiste

- Classe VII (9-10 ans) : molaires entièrement abrasées

Les schémas d'usure occlusale retenus pour l'attribution d'une dent isolée à un stade précis sont donnés dans la figure 2. Les fossettes persistant sur les deux lobes, la différenciation des stades II et III de la $M_{1}$ et des stades III et IV de la $M_{2}$ se fait biométriquement: un diagramme de dispersion du diamètre mésio-distal occlusal en fonction du diamètre vestibulo-lingual permettant d'isoler les deux stades. L'observation du degré d'usure de la surface occlusale de la $\mathrm{P}_{4}$ et de la $\mathrm{M}_{1}$, sans prise en compte de la hauteur de la couronne, ne permet pas une attribution au-delà du stade $\mathrm{V}$; pour la $\mathrm{M}_{2}$, la limite se situe au niveau du stade VI. Seule la $\mathrm{M}_{3}$ permet la différenciation des individus les plus âgés. Comme on peut le voir dans la figure 2, certains degrés d'usures ne peuvent être rattachés à un stade précis. C'est le cas notamment des $M_{1}$ présentant un lobe antérieur totalement usé et une fossette postérieure d'étendue limitée. D'après les séries dentaires, elles peuvent être rattachées soit au stade IV, soit au stade V. Afin d'attribuer ces dents à un stade précis, la procédure développée par S. Payne (1973) a été utilisée sur l'ensemble des séries dentaires. Ainsi, en prenant l'exemple de la $\mathrm{M}_{1}$, six séries sont caractérisées par le stade d'usure décrit ci-dessus : cinq d'entre elles, d'après les dents adjacentes, peuvent être rattachées au stade IV et une au stade V. Ainsi, pour un nombre $\mathrm{x}$ de $\mathrm{M}_{1}$ présentant cette usure, le nombre de $M_{1}$ attribuable au stade IV est de : $x^{*}(5 / 6)$ et pour le stade V : $\mathrm{x}^{*}(1 / 6)$. 
Figure 2 : Schémas de l'usure des surfaces occlusales retenus pour la détermination des classes d'âge des dents d'Antilope saïga (conventions : Payne 1973).

Figure 2 : Wear stages adopted for the determination of Saiga antelope age classes (codes : Payne 1973).

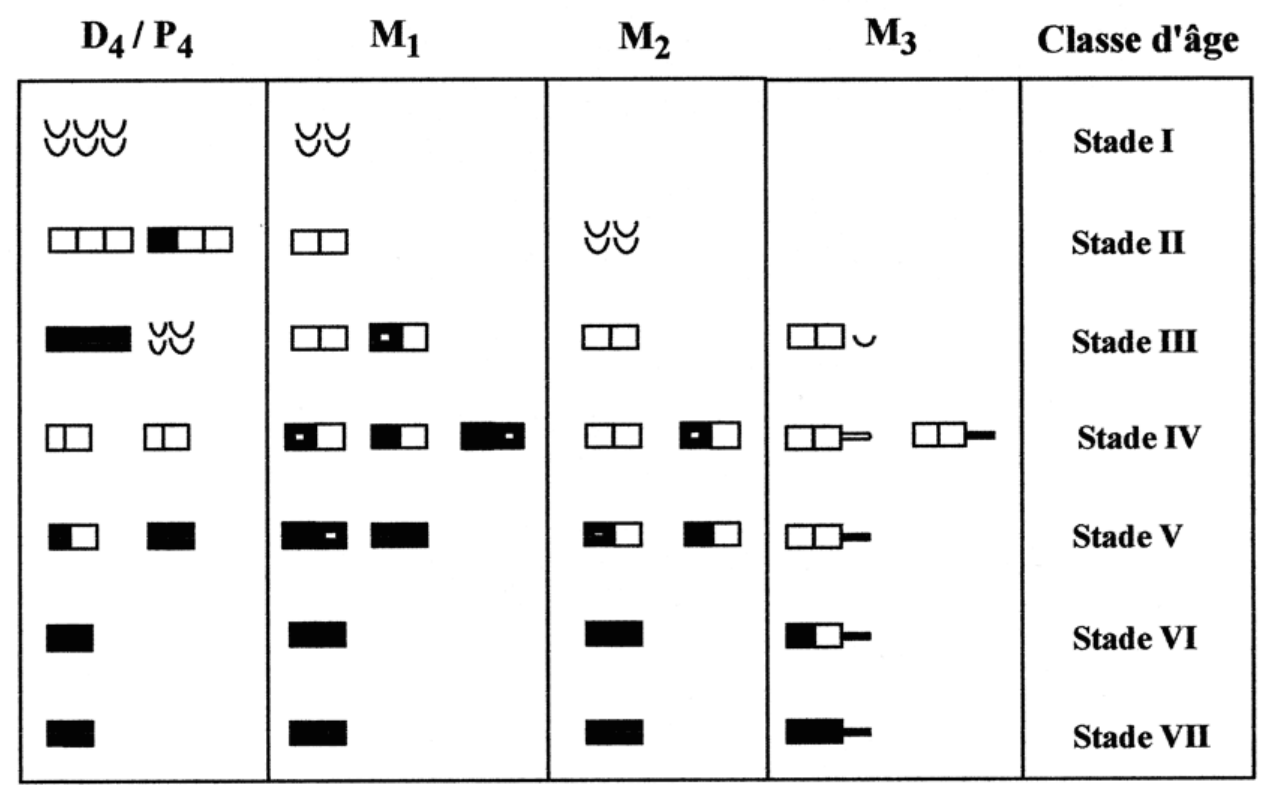

\section{Dimorphisme sexuel}

La détermination du sexe des animaux abattus est également importante pour la caractérisation des stratégies d'approvisionnement. La possibilité de distinguer le sexe des animaux présents dans les assemblages archéologiques passe soit par la reconnaissance d'attributs sexuels soit, pour des animaux présentant un dimorphisme sexuel, par la biométrie des éléments squelettiques (e. g. Altuna, 1978; Black, 1978 ; Delpech et Le Gall, 1983; Grigson, 1987; Larson et Taber, 1980; Mariezkurrena et Altuna, 1983 ; Noble et Crerar, 1993 ; Purdue, 1983 ; Steel, 1962 ; Todd, 1986).

L'Antilope saïga est souvent considérée comme une espèce présentant un faible dimorphisme sexuel. Seuls les attributs sexuels comme les cornes qui ne sont présentes que chez les mâles pourraient permettre une différenciation sexuelle. Cette méthode est peu utilisable en archéologie puisque de nombreuses chevilles osseuses ont pu être abandonnées sur les sites d'abattage en raison de leur intérêt nutritif très limité.

Afin d'estimer le nombre minimum de mâles et de femelles chassés dans les différents gisements magdaléniens, nous avons recherché si le dimorphisme sexuel était perceptible sur les os post-crâniens. À notre connaissance, ce type d'étude n'a jamais été mené sur l'Antilope saïga. Par conséquent, nous avons été contraintes d'utiliser les restes d'Antilope saïga provenant du site de Saint-Germain-la-Rivière. L'ensemble des extrémités d'os longs, les carpiens et les tarsiens ont été mesurés selon les critères décrits par J.-L. Guadelli (1987). Si un dimorphisme sexuel conséquent existe entre les mâles et les femelles, il devrait être possible de visualiser, sur les diagrammes de dispersion, deux populations distinctes.

30 L'absence de référentiel actuel est préjudiciable à cette étude puisqu'il n'y a aucun moyen de tester si les groupes mis en évidence sur les diagrammes de dispersion font réellement référence aux mâles et aux femelles. Dans le but d'établir une sorte de 
référentiel, le matériel provenant du Quéroy a donc été intégré à l'étude. Ce gisement, situé en Charente, est un aven-piège ayant livré une faune extrêmement bien conservée comprenant, en dehors de l'Antilope saïga, des ossements de Renne, de Bovinés, d'Equus caballus arcelini et d'Equus hydruntinus (Tournepiche 1982, 1996). Les antilopes saïga sont regroupées dans le niveau de base sur une surface de deux $\mathrm{m}^{2}$. Six squelettes ont été trouvés entremêlés : deux individus immatures et quatre adultes dont deux mâles et deux femelles.

Comme on peut le voir sur les figures 3 et 4 , les individus provenant du Quéroy sont systématiquement plus petits que ceux de Saint-Germain-la-Rivière ${ }^{1}$. Ils sont donc difficilement exploitables en tant que référentiel. Que ce soit pour l'astragale ou pour le calcanéum (qui sont les exemples les plus parlants), il est impossible de distinguer sur les diagrammes de dispersion les mâles des femelles: le nuage de points est continu. Cette impression est confirmée par les histogrammes qui ne présentent pas de distribution bimodale. L'absence de référentiel ne permet pas de savoir si les nuages de points sont de cette forme en raison de l'abattage systématique d'individus d'un sexe particulier ou en raison de l'absence de dimorphisme sexuel sur les os post-crâniens d'Antilope saïga, impliquant un chevauchement des nuages de points relatifs aux femelles et aux mâles.

Figure 3 : Taille des talus d'Antilope saïga de Saint-Germain-la-Rivière et du Quéroy. Figure 3 : Size of Saiga antelope talus from Saint-Germain-la-Rivière and Le Quéroy.
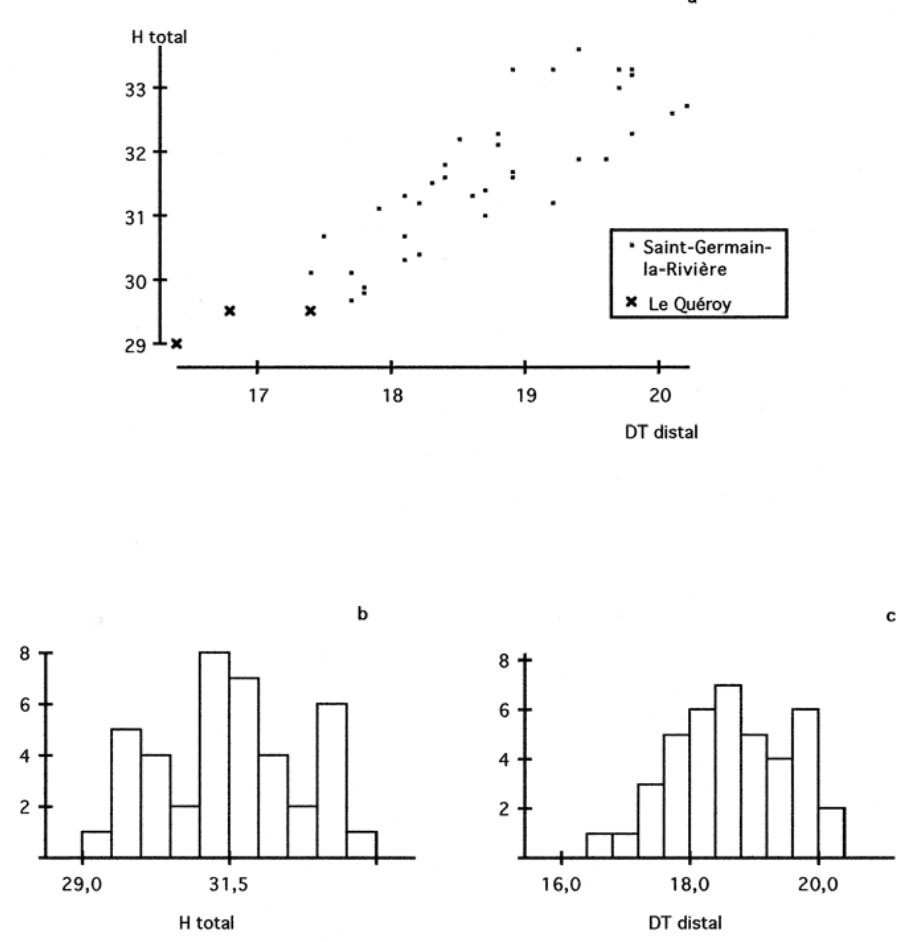

- a- diagramme de dispersion de la hauteur en fonction du diamètre transversal distal ; bhistogramme des hauteurs; c- histogramme des diamètres transversaux distaux (mesures en mm). - a-scatterplot of height by distal transversal diameter ; $b$ - heights histogram ; c-distal transversal diameters histogram (lengths in $\mathrm{mm}$ ). 
Figure 4 : Taille des calcanéums d'Antilope saïga de Saint-Germain-la-Rivière et du Quéroy. Figure 4 : Size of Saiga antelope calcaneum from Saint-Germain-la-Rivière and Le Quéroy.
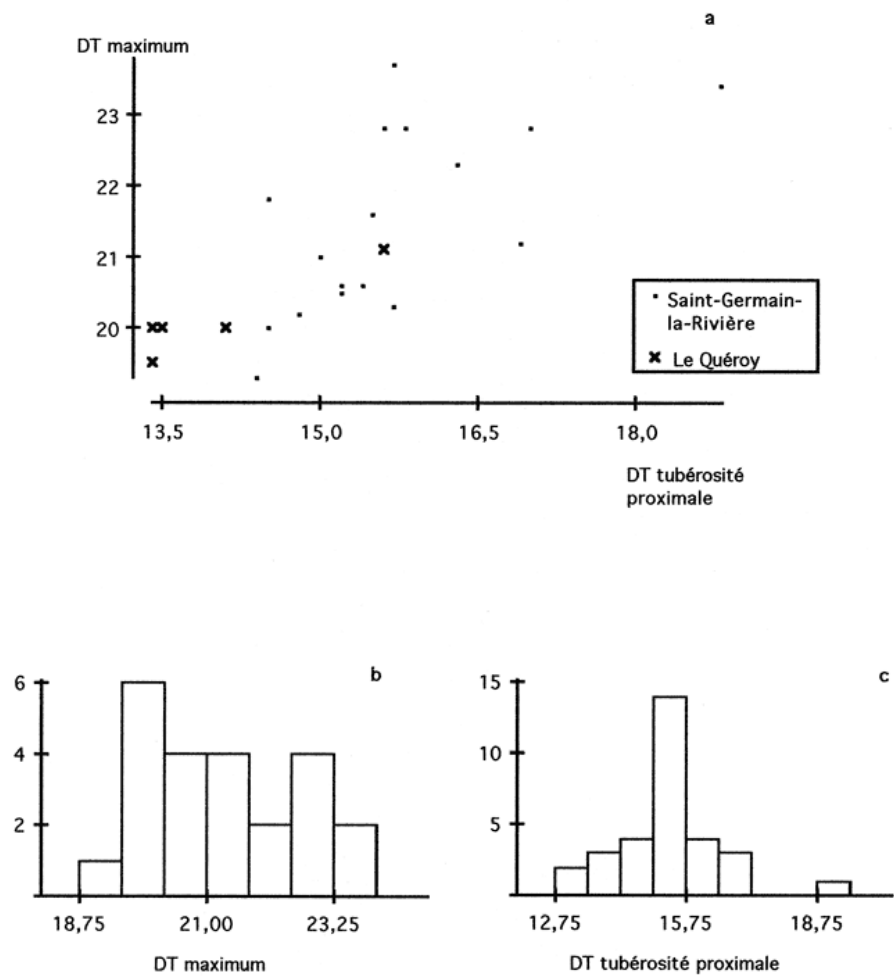

- a- diagramme de dispersion du diamètre transversal maximal en fonction du diamètre transversal de la tubérosité ; b- histogramme des diamètres transversaux maximaux; c- histogramme des diamètres transversaux des tubérosités proximales (mesures en $\mathrm{mm}$ ).

- a- scatterplot of maximum transversal diameter by transversal diameter of the tuberosity ; $b$ - maximum transversal diameters histogram ; c- transversal diameters of the tuberosity histogram (lengths in $\mathrm{mm}$ ). 
Figure 5 : Dimorphisme sexuel des premières phalanges d'Antilope saïga - Diagramme de dispersion de la longueur totale en fonction du diamètre transversal proximal et diagramme de dispersion du diamètre transversal en milieu de diaphyse en fonction de l'indice de robustesse. Figure 5 : Sexual dimorphism of Saiga antelope first phalanx - Scatterplot of total length by proximal transversal diameter and scatterplot of transversal diameter in the middle diaphysis by the robustness index (lengths in $\mathrm{mm}$ ).
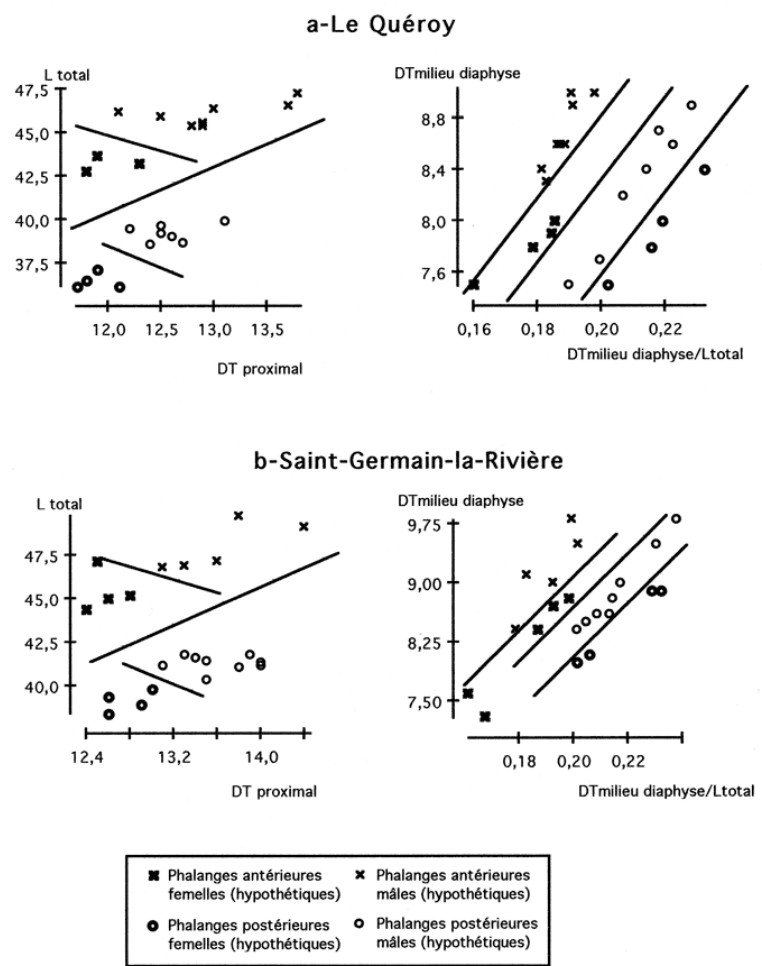

- a- Le Quéroy ; b- Saint-Germain-la-Rivière (mesures en mm).

Les premières phalanges sont les seuls éléments squelettiques pour lesquels la distinction mâle/femelle semble possible. Pour le Quéroy, si l'on examine le diamètre transversal du milieu de la "diaphyse" en fonction du degré de robustesse (Diamètre transversal du milieu de la "diaphyse" par rapport à la longueur de la phalange) ou la longueur de la phalange en fonction de son diamètre transversal proximal, il est possible de distinguer les phalanges antérieures (croix), des phalanges postérieures (cercles) : les premières étant plus longues et moins robustes que les secondes (fig. $5 \mathrm{a}$ ). $\mathrm{Au}$ sein de ces deux nuages de points, il semble possible de différencier deux sousgroupes qui pourraient correspondre aux mâles et aux femelles (pour un indice de robustesse similaire, les phalanges de femelles auraient un diamètre transversal en milieu de "diaphyse" systématiquement plus faible que celui des phalanges de mâles). Les diagrammes de dispersion obtenus à partir du matériel Saint-Germain-la-Rivière sont similaires à ceux du Quéroy (fig. 5b).

Pour confirmer ces résultats, il faudrait poursuivre l'étude en mesurant des squelettes d'Antilope saïga de sexe connu et vérifier si, effectivement, la taille des premières phalanges est réellement un facteur discriminant les mâles des femelles. L'examen d'un tel référentiel permettrait également de conclure si d'autres éléments ou portions squelettiques sont susceptibles d'être utilisés pour l'étude du dimorphisme sexuel. 


\section{Exploitation de l'Antilope saïga en Aquitaine}

\section{Une exploitation limitée dans le temps et dans l'espace}

Le tableau 2 répertorie l'ensemble des niveaux magdaléniens d'Aquitaine dont la faune a fait l'objet d'étude. Pour la discussion, la chronologie du Pléniglaciaire et du Tardiglaciaire présentée par M. Magny $(1995$, p. 48) a été utilisée. Le Magdalénien s'étend sur trois grandes périodes climatiques : le Pléniglaciaire (18 500 - $15000 \mathrm{BP})$, le Dryas ancien (15000 - 13000 BP) et le Bölling/Alleröd (13000 - 11000 BP). Les ensembles magdaléniens ayant fait l'objet de datations radiométriques ont été répartis dans ces trois ensembles chronologiques en fonction des dates obtenues. En l'absence de ces données, les cultures magdaléniennes ont été prises en compte. Ainsi, le Badegoulien et le Magdalénien ancien ont été attribués au Pléniglaciaire, le Magdalénien moyen au Dryas ancien et le Magdalénien supérieur et final au Bölling/ Alleröd.

Tableau 2 : Sites magdaléniens d'Aquitaine.

Table 2 : Magdalenian sites in Aquitaine.

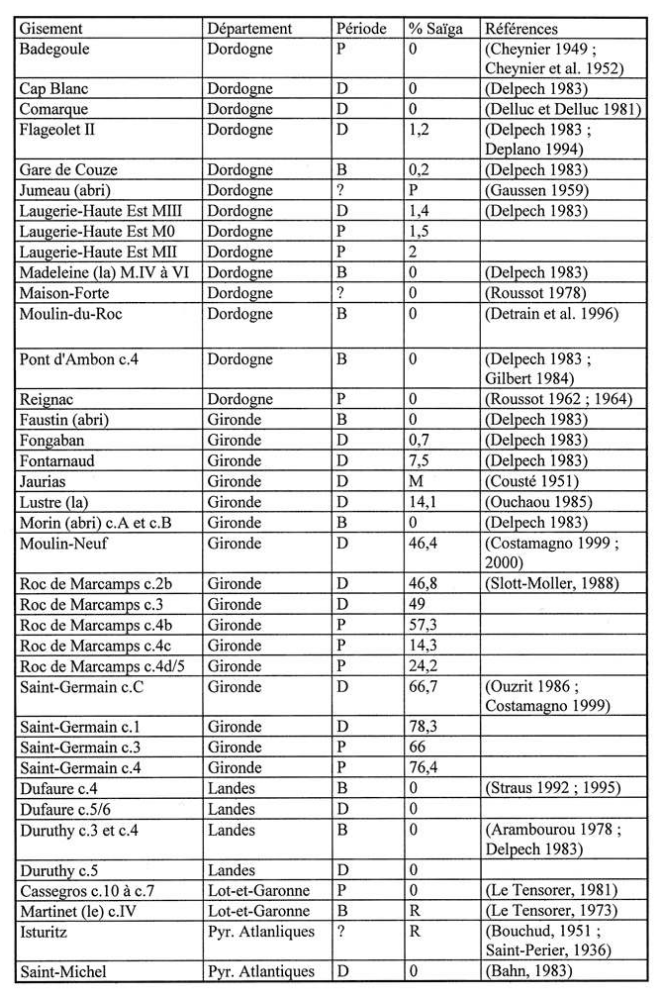

( $P$ : pléniglaciaire, $D$ : Dryas, $B$ : Bölling/Alleröd, $P$ : présente, $R$ : rare, $M$ : majoritaire).

( $P$ : pléniglaciaire, $D$ : Dryas, $B$ : Bölling/Alleröd, $P$ : present, $R$ : rare, $M$ : abundant).

L'examen du tableau 2 montre que l'Antilope saïga est présente dans toute l'Aquitaine (à l'exception des Landes) durant le dernier Pléniglaciaire et le Dryas ancien. A partir du Bölling, on ne la trouve plus qu'en Dordogne et dans le Lot-et-Garonne. En effet, comme le signale F. Delpech (1989), dès cette période, elle commence à quitter les plaines d'Aquitaine. Durant le dernier Pléniglaciaire et le Dryas ancien, bien que présente dans toute l'Aquitaine, l'Antilope saïga n'est exploitée intensivement qu'en Gironde. D'une part, tous les gisements girondins appartenant à ces deux périodes 
contiennent des ossements d'Antilope saïga. D'autre part, la Gironde est la seule zone d'Aquitaine ayant livré des ensembles osseux magdaléniens dominés par cette espèce. Comme nous allons le voir, l'exploitation intense de l'Antilope saïga en Gironde au cours du dernier Pléniglaciaire et du Dryas ancien est le reflet de son abondance dans l'environnement plutôt que d'un choix délibéré des magdaléniens en faveur de cette espèce.

\section{Les exemples de Saint-Germain-la-Rivière et de Moulin-Neuf}

Ces deux gisements sont tous deux situés en Gironde. Le site de Saint-Germain-laRivière découvert en 1929 a fait l'objet de plusieurs campagnes de fouilles. Le matériel étudié provient des fouilles réalisées par G. Trécolle dans les années 60 dans le talus prolongeant le grand abri. Ce talus fouillé sur une surface de $8 \mathrm{~m} 2$ a livré une séquence stratigraphique au sein de laquelle cinq niveaux ont été différenciés : un ensemble inférieur comportant les couches 4 (GIF 5479:16 $200 \pm 600 \mathrm{BP}$; OXA/LYON-617 : $16890 \pm$ $130 \mathrm{BP}$ ) et 3 et un ensemble supérieur comprenant les couches 1 (OXA/LYON-615 : $15330 \pm 150 \mathrm{BP}$ ) et C (GIF : $14100 \pm 160 \mathrm{BP}$ ), la couche 2 se situant à l'interface de ces deux ensembles. Pour M. Lenoir (1983), l'industrie lithique de l'ensemble inférieur caractérisé par un outillage assez grossier, riche en éclats pourrait être attribué à du Magdalénien ancien tandis que l'ensemble supérieur aux industries beaucoup plus laminaires serait du Magdalénien moyen.

Le site de Moulin-Neuf (Saint Quentin de Baron) découvert en 1939 a également fait l'objet de fouilles multiples. Les collections étudiées proviennent des fouilles réalisées par M. Lenoir dans l'abri principal. Les ossements appartiennent à un seul niveau : la couche 2 attribuée au Magdalénien moyen (Ly 2352:13 570 \pm 260 BP ; Ly 2275: $14280 \pm$ $440 \mathrm{BP}$ ) ( $c f$. Lenoir 1983 pour un historique plus précis de ces deux gisements).

\section{Importance de l'Antilope saïga dans l'alimentation des hommes ayant occupé Saint-Germain-la-Rivière ${ }^{2}$ et Moulin-Neuf}

Les spectres fauniques de ces deux gisements sont relativement diversifiés. A SaintGermain-la-Rivière, la richesse taxonomique dans les couches 3 et 1 est respectivement de neuf et huit tandis qu'à Moulin-Neuf, elle est de onze (tabl.3). Seuls les taxons présentant des traces anthropiques ont une origine humaine attestée (Costamagno 1999). Ainsi, à Saint-Germain-la-Rivière, cinq taxons ont incontestablement été chassés par l'Homme: l'Antilope saïga, le Renne, le Cheval, les grands Bovidés et Equus hydruntinus. A l'exception de cette dernière espèce, les mêmes taxons ont été exploités à Moulin-Neuf (Costamagno 2000). Comme on peut le voir dans le tableau 3, dans ces deux gisements quelle que soit la couche considérée, l'Antilope saïga est l'espèce majoritairement chassée. Cependant, si à Saint-Germain-la-rivière, l'Antilope saïga fournit la majeure partie des ressources carnées en particulier dans la couche 1 ( $78,3 \%$ et inverse de l'indice de Simpson $\left.{ }^{3}: 1,606\right)$, à Moulin-Neuf, la part du Cheval dans l'alimentation n'est pas négligeable ( $25,6 \%$ contre $46,4 \%$ pour l'Antilope saïga, indice de Simpson: 2,892). Etant donné, le faible poids d'une carcasse d'Antilope saïga (maximum $40 \mathrm{~kg}$ ) par rapport à une carcasse de Cheval (300-350 kg), il est probable qu'à Moulin-Neuf, l'Antilope saïga ne constituait qu'une source carnée secondaire, le Cheval étant l'espèce principale. 
Tableau 3 : Espèces présentes dans les ensembles osseux de Saint-Germain-la-Rivière et de Moulin-Neuf.

Table 3 : Taxonomic composition of Saint-Germain-la-Rivière and Moulin-Neuf bone assemblages.

\begin{tabular}{|l|r|r|r|r|r|r|}
\hline \multirow{2}{*}{} & \multicolumn{3}{|c|}{ Saint-Germain } & \multicolumn{2}{c|}{ Moulin-Neuf } \\
\cline { 2 - 7 } & \multicolumn{2}{|c|}{ Couche 1 } & \multicolumn{2}{c|}{ Couche 3 } & \multicolumn{2}{c|}{ Couche 2 } \\
\cline { 2 - 7 } & NISP & MNI & NISP & MNI & NISP & MNI \\
\hline Panthera spelaea & - & - & & & 1 & 1 \\
\hline Canis lupus & 1 & 1 & 1 & 1 & 4 & 1 \\
\hline Vulpes/Alopex & 1 & 1 & 4 & 1 & 42 & 2 \\
\hline Meles meles & - & - & - & - & 3 & 1 \\
\hline Sus scrofa & - & - & & & 1 & 1 \\
\hline Cervus elaphus & - & - & 2 & 1 & 2 & 1 \\
\hline Rangifer tarandus & 298 & 3 & 264 & 3 & 321 & 5 \\
\hline Bovinae & 60 & 2 & 90 & 3 & 168 & 3 \\
\hline Rupicapra rupicapra & - & - & & & & -1 \\
\hline Saiga tatarica & 1742 & 17 & 1011 & 16 & 911 & 18 \\
\hline Equus caballus & 114 & 3 & 154 & 5 & 502 & 8 \\
\hline Equus hydruntinus & 7 & 1 & 1 & 1 & & - \\
\hline Leporidae & 2 & 1 & 4 & 1 & 7 & 1 \\
\hline Total & $\mathbf{2 2 2 5}$ & $\mathbf{2 9}$ & $\mathbf{1 5 3 1}$ & $\mathbf{3 2}$ & $\mathbf{1 9 6 2}$ & $\mathbf{4 2}$ \\
\hline
\end{tabular}

Saison(s) de chasse

A Saint-Germain-la-Rivière, les saisons de chasse à l'Antilope saïga ont été documentées grâce à des études cémentochronologiques menées par E. Pubert (Costamagno 1999). A Moulin-Neuf, de telles études n'ont pu être menées en raison de la fragmentation des molaires d'Antilope saïga. Les données reposent donc sur les stades d'éruption dentaire (Costamagno 2000).

Comme on peut le voir sur la figure 6, dans la couche 3 de Saint-Germain-la-Rivière les antilopes saïga ont été chassées essentiellement durant la fin de la mauvaise et le début de la bonne saison. Dans la couche 1, la chasse à l'Antilope semble se prolonger puisque des animaux ont été abattus de la fin de la mauvaise saison à la fin de la bonne saison, le pic se situant au début de la bonne saison. 
Figure 6 : Saison d'abattage des antilopes saïga à Saint-Germain-la-Rivière. Figure 6 : Saiga antelope killing season in Saint-Germain-la-Rivière.
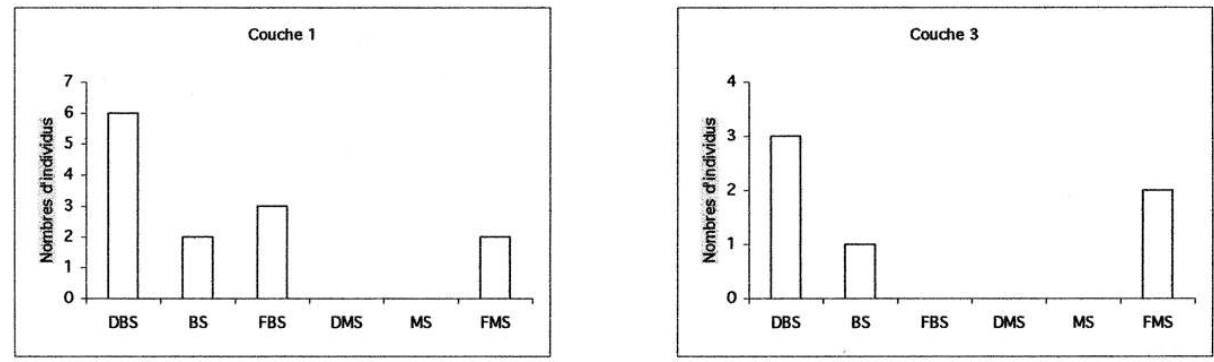

- a- couche 1 ; b- couche 3 (DBS : début de bonne saison, BS : bonne saison, FBS : fin de bonne saison, DMS : début de mauvaise saison, MS : mauvaise saison ; FMS : fin de mauvaise saison). - a-Level 7 ; b- level 3.

Dans les deux niveaux, l'absence d'antilope tuée durant la mauvaise saison peut indiquer l'abandon de cette zone par l'Homme préhistorique au cours de cette période de l'année, l'exploitation d'autres ressources, au cours de la mauvaise saison, étant une autre hypothèse à envisager. En tout état de cause, que ce soit en termes de mobilité ou en termes de diversification des sources d'approvisionnement, les choix humains étaient une réponse à la disparition de l'Antilope saïga de l'environnement local de Saint-Germain-la-Rivière, durant la mauvaise saison. Cette hypothèse est soutenue par l'éthologie des antilopes saïga actuelles. Même s'il est vrai que, durant les années peu rigoureuses, l'Antilope saïga peut rester sédentaire, les migrations d'hiver vers des zones moins enneigées sont un comportement retrouvé fréquemment chez cette espèce. Par analogie, l'absence de restes d'Antilope saïga durant la mauvaise saison montre que la zone environnant Saint-Germain-la-Rivière peut être assimilée à l'aire de répartition d'été de l'espèce, dans cette aire géographique. Durant l'hiver, les troupeaux migraient probablement vers le Sud ou l'Ouest. Dans la couche 3, les animaux n'ont été abattus qu'à la fin de la mauvaise et au début de la bonne saison. Les datations radiométriques montrent que les niveaux de base se sont déposés juste après le dernier maximum glaciaire. L'accroissement de la sécheresse, durant cette période, pourrait expliquer l'absence d'Antilope tuée durant l'été: ce territoire ne correspondant plus à l'aire de répartition d'été mais à une zone de passage vers des pâturages d'été situés probablement plus au Nord. De telles migrations d'été sont actuellement connues (cf. supra).

Face à la diminution des troupeaux d'antilopes saïga, les hommes semblent avoir opté pour une diversification des ressources exploitées plutôt que pour l'abandon de la région. Les études de saisonnalité effectuées sur les dents de chevaux (Burke 1995) et de rennes (Gordon 1988), bien qu'à prendre avec précautions en raison des effectifs étudiés limités, apportent des renseignements utiles sur les stratégies d'approvisionnement de ces hommes. Dans la couche 3, ces résultats indiquent une exploitation intense de l'Antilope saïga au début de la bonne saison puis une diversification progressive des ressources, liée à l'absence de cette dernière de la région, en faveur notamment du Cheval durant l'été (Burke 1995) et du Renne durant l'hiver (Gordon 1988). Dans la couche 1, les données sont moins concluantes que dans la couche 3 en raison du nombre extrêmement limité d'observations : la seule dent de cheval lisible indique un animal tué en hiver (Burke 1995). Ceci pourrait indiquer une réaction identique des hommes face à la migration des antilopes : la nécessité de pallier 
l'absence de ces troupeaux se faisant ressentir plus tardivement en raison de l'exploitation, par l'Antilope saïga, des pâturages environnant Saint-Germain-la-Rivière durant la totalité de la bonne saison.

A Moulin-Neuf, définir la saison d'abattage des antilopes saïga est plus problématique. D'après les premières incisives déciduales, trois individus ont moins d'un an (remplacement de ces dents par des définitives entre le treizième et le quatorzième mois (Bannikov 1967)). En outre, les quatrièmes déciduales supérieures provenant d'un minimum de trois individus sont très usées et indiquent donc un âge proche de quinze mois. Si on part du principe que les premières incisives déciduales et les quatrièmes déciduales supérieures proviennent des mêmes individus, on peut en déduire que ces animaux ont été tués entre leur dixième et treizième mois. Les antilopes saïga naissant de fin avril à la mi-mai, ces individus ont pu être abattus entre février et juin. L'absence de dents déciduales peu usées peut indiquer :

1. une exploitation saisonnière des antilopes saïga de février à juin,

2. le refus d'abattre des individus très jeunes (de moins de neuf mois),

3. un problème taphonomique: destruction différentielle des dents provenant de jeunes individus (Guadelli et Ozouf 1994 ; Texier et al. 1998).

Moulin-Neuf étant contemporain de la couche 1 de Saint-Germain-la-Rivière, on peut tout à fait imaginer une exploitation saisonnière de l'Antilope saïga de la fin de la mauvaise saison à la fin de la bonne saison, l'hypothèse 2 ou 3 étant alors à privilégier.

\section{Recrutement des Antilopes saïga et stratégies de chasse}

A Saint-Germain-la-Rivière comme à Moulin-Neuf, nous avons utilisé la méthode présentée ci-dessus pour estimer l'âge des antilopes saïga (Costamagno 1999). Pour chaque ensemble osseux, la forme des profils de mortalité est ensuite documentée à l'aide des diagrammes ternaires définis par M.C. Stiner (1990, 1991a), modifiés par S. Costamagno (1999). Pour évaluer le sexe des animaux abattus, la taille des premières phalanges a été étudiée.

Au Magdalénien moyen que ce soit à Saint-Germain-la-Rivière (couche 1) ou à MoulinNeuf, les profils de mortalité sont similaires et correspondent à des profils de mortalité catastrophique (fig. 7). Cependant, la présence d'individus d'âges différents au sein de la première année, ainsi que l'échelonnement, sur une grande partie de l'année, de la chasse à l'Antilope saïga permettent de rejeter l'hypothèse d'une mort en masse des individus. Les courbes de mortalité montrent donc une exploitation opportuniste des ressources, les animaux étant prélevés au hasard au sein d'une population vivante stable. A Saint-Germain-la-Rivière, les profils de mortalité mettent en évidence un changement au niveau du recrutement des individus, au cours du temps. En effet, dans la couche 3, les animaux les plus faibles sont préférentiellement chassés: les jeunes mais surtout les vieux adultes dominent largement l'ensemble osseux (fig. 7) . Ces différences sont difficiles à expliquer. Plusieurs hypothèses peuvent être évoquées :

1. l'évolution des techniques permettant aux Hommes du Magdalénien supérieur de tuer plus facilement les individus adultes,

2. la chasse de groupes constitués majoritairement de vieux adultes dans l'ensemble inférieur,

3. l'abattage des individus les plus faibles dans l'ensemble inférieur afin d'éviter une surexploitation des ressources et, ainsi, pallier une diminution conséquente des effectifs des troupeaux chassés, 
4. l'abattage des vieux individus dans l'ensemble inférieur pour des besoins particuliers,

5. la recherche, dans l'ensemble inférieur, d'un gain de temps se traduisant par la traque d'animaux plus faciles à chasser.

Des cinq hypothèses formulées, la cinquième paraît la plus plausible ( $c f$. discussion dans Costamagno 1999 : 233-234).

Figure 7 : Profils de mortalité des antilopes saïga dans les couches 1 et 3 de Saint-Germain-laRivière et dans le niveau 2 de Moulin-Neuf (MN) (en U : courbe de mortalité normale, en $\mathrm{L}$ : courbe de mortalité catastrophique)

Figure 7: Saiga antelope mortality profiles in the levels 1 and 3 of Saint-Germain-la-Rivière and in the level 2 of Moulin-Neuf (MN) (en U : attritional mortality profile, en L : catastrophic mortality profile).

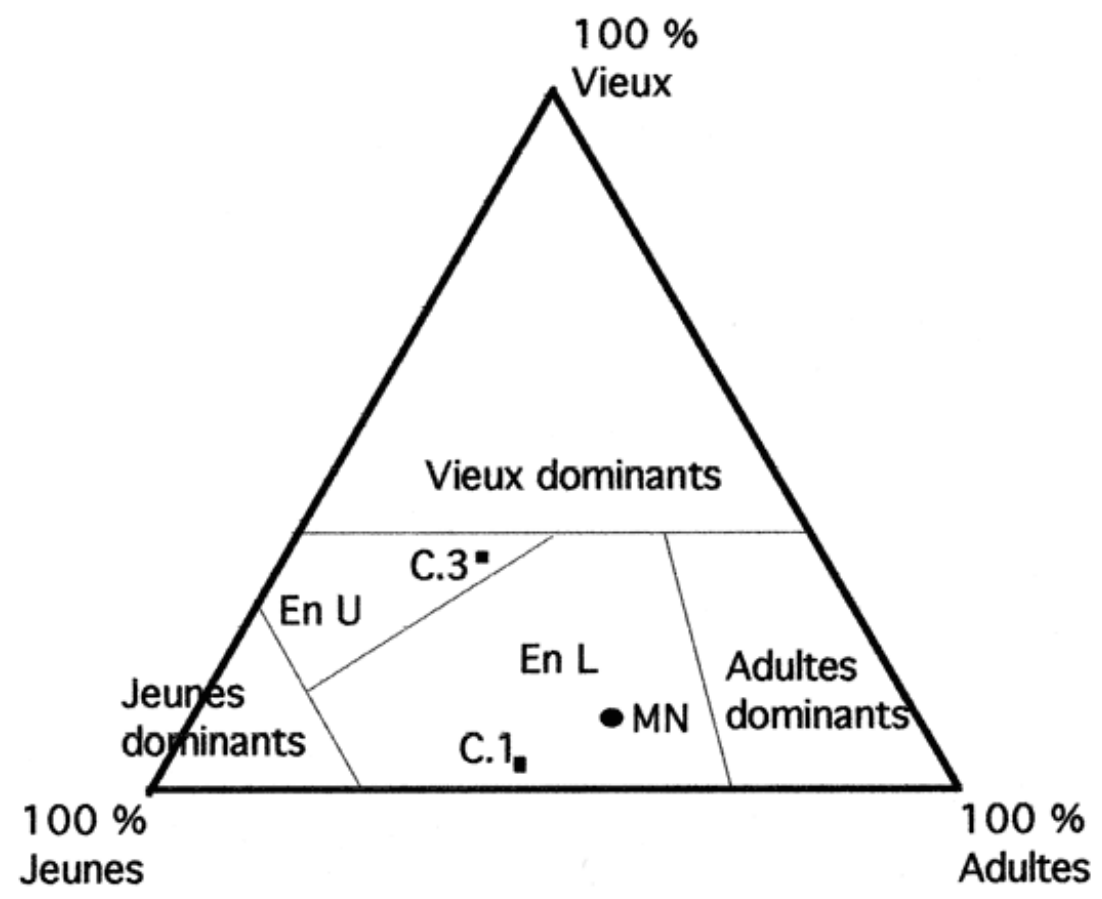

En ce qui concerne le sexe des individus abattus, comme nous l'avons vu, seule la taille des phalanges permet de mettre en évidence le dimorphisme sexuel des antilopes saïga. Par conséquent, il est impossible d'estimer les nombres minimums de mâles et de femelles dans chaque couche, chaque individu possédant huit phalanges. Dans les deux gisements, des mâles et des femelles semblent avoir été chassés par les hommes parmi les hardes mixtes, tous deux étant durant cette période dans la même condition physique.

A Moulin-Neuf ainsi qu'à Saint-Germain-la-Rivière, l'âge des antilopes saïga abattues montre une stratégie de chasse non sélective. L'abondance de l'Antilope saïga semble reflèter une vision saisonnière de l'environnement local plutôt qu'un recrutement préférentiel de cette espèce par les hommes. Cette hypothèse est étayée par la petite taille de cet animal. Il existe une relation entre le poids de l'animal et le nombre de calories qu'il peut fournir (Simms 1987). De ce fait, l'abondance de l'Antilope saïga est liée à sa profusion dans le milieu environnant et/ou à sa traque aisée : les chasseurs étant plus préoccupés par la taille du gibier chassé que par des affinités taxonomiques (Broughton 1994). 


\section{Transport et traitement des carcasses d'Antilope saïga} différentielle des éléments squelettiques (e.g. Binford 1978, 1981; Farizy et al. 1994; O'Connell et al. 1990 ; Perkins et Daly 1968; Stiner 1994; Thomas et Mayer 1983). Cependant, la forme des profils squelettiques est dépendante de nombreux processus taphonomiques. En effet, l'absence d'un élément squelettique sur un site peut être liée aux stratégies de transport adoptées par les hommes (Bartram 1993b ; Binford 1978; Speth 1983), à un problème de conservation différentielle (Brain 1981; Lyman 1984 ; Marean et al. 1992), à des méthodes d'analyse particulièrs (Bartram 1993a ; Bartram et Marean 1999; Marean 1998; Marean et Frey 1997) ou bien encore à des pertes de matériel intervenues durant le stockage (Costamagno soumis-a). taphonomiques sur les profils squelettiques d'Antilope saïga a été évalué (Costamagno 1999, 2000). En ce qui concerne plus particulièrement le transport, il apparaît que les carcasses d'Antilope saïga étaient majoritairement transportées entières sur les deux sites (fig. 8). La sous-représentation du squelette axial semble liée à un problème de conservation différentielle. L'absence de corrélation entre les indices d'utilité et la représentation des éléments squelettiques est bien compatible avec l'économie opportuniste mise en évidence à partir du recrutement des individus.

Figure 8 : Représentation des éléments squelettiques d'Antilope saïga en MAU.

Figure 8 : Saiga antelope skeletal part profiles in MAU.

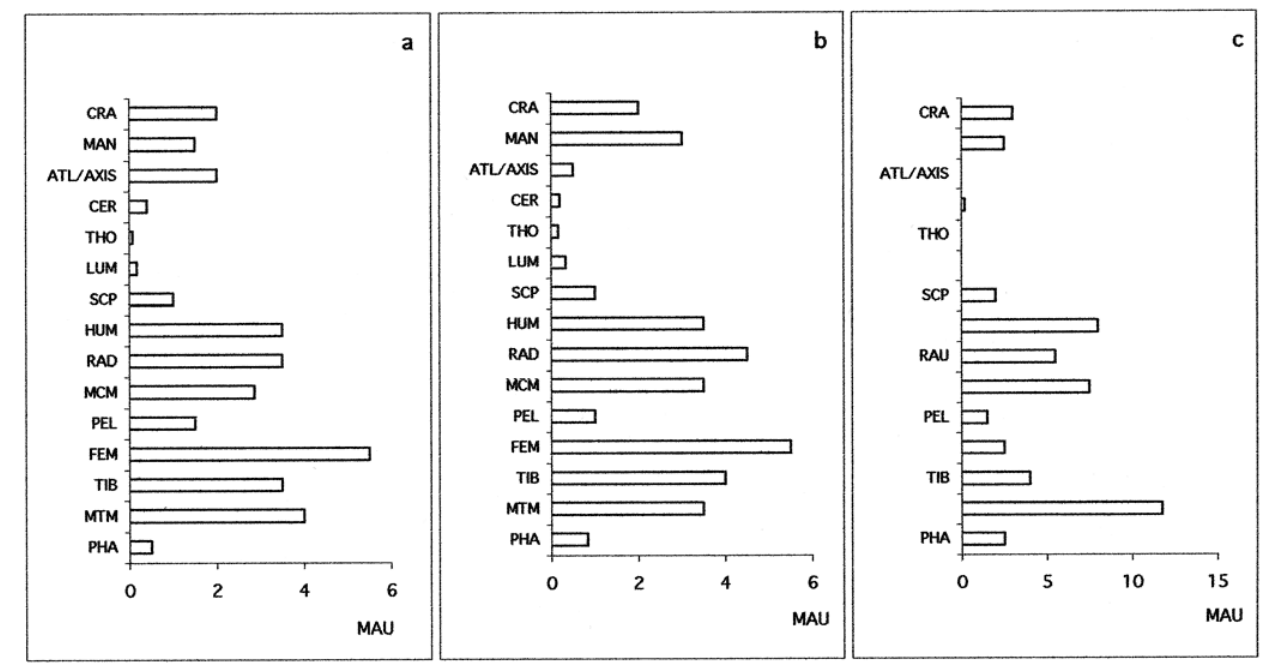

- a- dans le carré M21 de la couche 1 de Saint-Germain-la-Rivière ; b- dans le carré L21 de la couche 3 de Saint-Germain-la-Rivière ; c- dans la couche 2 de Moulin-Neuf (CRA : crâne, MAN : mandibule, ATL/AXIS : atlas/axis, CER : vertèbres cervicales, THO : vertèbres thoraciques, LUM : vertèbres lombaires, SCP : scapula ; HUM : humérus ; RAD : radius ; MCM : métacarpe ; PEL : pelvis, FEM : fémur ; TIB : tibia ; MTM : métatarse ; PHA : phalanges).

- $a$ - in square M21 of the level 1 of Saint-Germain-la-Rivière ; $b$ - in square $L 21$ of the level 3 of SaintGermain-la-Rivière; c- in the level 2 of Moulin-Neuf (CRA : skull, MAN : mandible, ATL/AXIS : atlas/axis, CER cervical vertebrae, THO : thoracic vertebrae, LUM : lumbar vertebrae, SCP : scapula; HUM : humerus; RAD : radius ; MCM : metacarpal ; PEL : pelvis, FEM : femur ; TIB : tibia ; MTM : metatarsal ; PHA : phalanxes).

Le traitement des carcasses d'Antilope saïga apparaît relativement similaire à MoulinNeuf et à Saint-Germain-la-Rivière. Les carcasses d'antilopes ont été intensément 
désarticulées mais également décharnées comme l'indique l'abondance des stries de boucherie sur les fragments diaphysaires d'os longs. En raison de la saison d'abattage, le décharnement des os longs semble lié à l'exploitation de la moelle et non pas au stockage des filets de viande (Costamagno, 1999). L'intense fragmentation des os longs mais également des mandibules et des phalanges indique, en outre, une exploitation maximale des ressources.

Il apparaît donc que les hommes qui ont occupé Saint-Germain-la-Rivière et MoulinNeuf pratiquaient une chasse non sélective saisonnière sur des hardes mixtes d'Antilope saïga. Les carcasses ainsi obtenues étaient ensuite transportées entières au camp puis intensivement traitées.

\section{Discussion}

D'après les résultats obtenus à Saint-Germain-la-Rivière et Moulin-Neuf, en Gironde, durant le dernier Pléniglaciaire et le Dryas ancien, l'Antilope saïga ne fournissait des ressources alimentaires que de façon saisonnière (entre la fin de la mauvaise saison et la bonne saison). Cette exploitation limitée dans l'année était liée à son absence de la région; cette zone correspondait alors à son territoire d'été. Un faisceau de preuves indique que, à Moulin-Neuf ou à Saint-Germain-la-Rivière, les hommes chassaient l'Antilope saïga à petite échelle. En effet, des chasses collectives à grande échelle impliquent la mort en masse d'individus. L'abattage simultané d'un grand nombre d'animaux au cours d'un épisode de chasse se traduit alors par une sélection et une exploitation des carcasses et des portions squelettiques les plus riches (Binford 1978; Speth 1983 ; Wheat 1972). Or, à Saint-Germain-la-Rivière comme à Moulin-Neuf, il n'y a aucune relation entre l'abondance des éléments squelettiques et leur utilité nutritive, les carcasses ayant été transportées entières depuis le site d'abattage au camp. L'exploitation maximale des ressources alimentaires provenant des carcasses d'Antilope saïga est un autre argument en faveur de chasses à petite échelle. La saison d'abattage des antilopes vient d'ailleurs conforter cette hypothèse. En effet, la finalité des grandes chasses collectives est de constituer des réserves de nourriture pour l'hiver. Au printemps comme en été qui sont les saisons d'abattage représentées à Saint-Germain-la-Rivière et à Moulin-Neuf, plusieurs facteurs vont à l'encontre de telles pratiques. En effet, au printemps, les troupeaux d'Antilope saïga ont une taille importante, mais les individus sont en mauvaise condition physique et l'été arrivant, avec ses fortes températures et la prolifération des mouches, empêche la conservation des denrées jusqu'à l'hiver. Durant l'été, les animaux commencent déjà à constituer leur réserve de graisse pour l'hiver, mais la petite taille des troupeaux (moins de 20 individus) empêche la mise en œuvre de grandes chasses collectives. En outre, la pratique de chasses à petite échelle n'implique pas l'attention de tous les membres du groupe qui peuvent alors s'appliquer à rechercher des ressources autres que carnées (Driver 1990). De telles stratégies sont beaucoup plus rentables que les grandes chasses collectives, notamment au printemps et en été, lorsque les sources exploitables sont très diversifiées. Pour l'Antilope saïga comme pour le Renne (Albrecht 1984; Binford 1978), les migrations d'automne semblent la période la plus favorable à la mise en pratique de ces types de chasse: les troupeaux étant importants, les animaux présentant leur condition physique optimale et l'approche de l'hiver permettant une conservation aisée, par congélation, des denrées périssables (Driver 1990). On peut 
imaginer, à cette période de l'année, la mise en œuvre de chasses collectives planifiées visant à tuer un grand nombre d'individus pour créer des réserves pour l'hiver, ces chasses prenant place à d'autres emplacements que Saint-Germain-la-Rivière ou Moulin-Neuf. Cependant, dans l'état actuel des données, aucun site de Gironde ne permet d'illustrer la pratique de telles chasses sur l'Antilope saigga. Seul l'ensemble osseux de Fongaban dominé à plus de $98 \%$ par du Bison (Delpech 1972) pourrait indiquer que les chasseurs-cueilleurs ayant vécu en Gironde pratiquaient, à certaines périodes de l'année, des chasses à grande échelle (Costamagno soumis-b). Malheureusement, l'absence d'étude archéozoologique (saison d'abattage, recrutement des individus, traitement des carcasses) ne permet pas de vérifier la validité de cette hypothèse.

En dehors de son influence sur les stratégies de chasse adoptées par les hommes, l'exploitation saisonnière de l'Antilope saïga a eu forcément des répercussions importantes sur le mode de vie des chasseurs-cueilleurs qui occupaient cette région. Face à la disparition des troupeaux d'Antilope saïga de la région, deux hypothèses sont envisageables :

1. les hommes suivent les troupeaux d'Antilope saïga et abandonnent la région,

2. ils exploitent de nouvelles ressources.

D'après les études menées à Saint-Germain-la-Rivière, à Moulin-Neuf mais également au Roc-de-Marcamps, les hommes ayant occupé la Gironde durant le dernier Pléniglaciaire et le Dryas ancien semblent avoir opté pour une diversification des ressources exploitées plutôt que pour l'abandon de la région. En effet, sur ces gisements, des animaux ont été abattus à toutes les périodes de l'année (Burke 1995; Costamagno 1999, 2000 ; Gordon 1988; Slott-Moller 1988). Durant cette période, la région semble donc avoir été occupée annuellement par des groupes de chasseurscueilleurs. L'utilisation quasi-exclusive du silex sénonien provenant des alluvions de la basse vallée de la Dordogne proche (Lenoir 1996) indique l'exploitation de territoires relativement restreints. Bien que des matières premières allochtones provenant du Périgord mais également du Piémont pyrénéen (chalosse) aient été signalées dans des ensembles magdaléniens (Lenoir et al. 1997), il apparaît que l'ensemble des ressources présentes dans cette zone géographique devait être suffisante pour subvenir aux besoins annuels des groupes de chasseurs-cueilleurs y vivant. Dans quelle mesure l'abondance de l'Antilope saïga dans la région, au printemps et en été, influait-elle sur les modalités d'exploitation des territoires de ces groupes? Dans l'état actuel des données, il est extrêmement délicat de répondre à cette question. Cette zone géographique par rapport à d'autres [Aude (Fontana 1998b, 1999), Haute-vallée de la Loire (Costamagno 1999 ; Fontana 1998a)] apparaît tout à fait originale en raison d'une fréquentation annuelle et continue. Cependant, la Gironde n'est pas la seule région à avoir été occupée annuellement par des groupes magdaléniens : en Dordogne (Burke 1995 ; Deplano 1994) ou bien encore dans les Pyrénées (Pailhaugue 1998), des animaux ont été abattus à toutes les périodes de l'année. Or, dans ces zones géographiques, l'Antilope saïga n'est que sporadique voire absente. En Gironde, ce sont donc les caractéristiques générales de la région qui semblent être à l'origine de cette occupation annuelle et continue. Seule l'étude archéozoologique des ensembles osseux plus récents de l'abri Faustin et de l'abri Morin dépourvus d'Antilope saïga (Delpech 1971, 1983) pourrait permettre d'évaluer réellement l'influence de cette espèce sur les modalités d'exploitation des territoires. 


\section{Conclusion} saïga en Gironde avait influé sur les stratégies de chasse des Magdaléniens. Outre les données archéozoologiques obtenues à Saint-Germain-la-Rivière et Moulin-Neuf, la connaissance de l'écologie et de l'éthologie de l'Antilope saïga s'est avérée essentielle pour comprendre les choix des Magdaléniens. En effet, s'il est vrai que l'introduction de carcasses entières d'Antilope saïga au camp ainsi que leur traitement intensif plaident en faveur de chasse à petite échelle, la prise en compte de l'écologie de cet animal permet d'apprécier les facteurs qui ont guidé les choix stratégiques des Magdaléniens. C'est également la connaissance des périodes de migration des antilopes saïga actuelles qui permet de conclure que la Gironde, durant le Dryas ancien, correspondait probablement aux territoires d'été de l'Antilope saïga. L'exploitation saisonnière de cette espèce mise en évidence à Saint-Germain-la-Rivière et à Moulin-Neuf est donc liée à l'absence de l'Antilope saïga de la région durant la mauvaise saison et non pas à un choix délibéré des chasseurs-cueilleurs. Au lieu de suivre les troupeaux d'antilopes saïga, il apparaît clairement que les Magdaléniens de Gironde ont choisi d'occuper annuellement cette région en diversifiant leurs ressources (Cheval, Renne). Dans quelle mesure, l'écologie de l'Antilope saïga a influé sur ce choix ? L'interrogation demeure...

\section{BIBLIOGRAPHIE}

ALBRECHT G. 1984 - Intensive fall hunting at Petersfels during Magdalenian : Questions concerning the motives. In : K. Kerke, J. Hahn et C.-J. Kind (Eds.), Jungpalaolithische Siedlungsstrukturen in Europa, p. 99-102. Tübingen, Archaeologica Venatoria.

ALTUNA J. 1978 - Dimorphisme sexuel dans le squelette postcéphalique de Capra pyrenaica pendant le Würm final. Munibe, 1978, 30, p. 201-214.

ALTUNA J. et MARIEZKURRENA K. 1996 - Primer hallazgo de restos oseos de antilope Saiga (Saiga tatarica L) en la Peninsula Iberica. Munibe, 1996, 48, p. 3-6.

ALVARD M. et KAPLAN H. 1991 - Procurement technology and prey mortality among indigenous neotropical hunters. In : M.C. Stiner (Ed.), Human predators and prey mortality, p. 80-103. Boulder, Westview press.

ARAMBOUROU R. (dir.) 1978 - Le gisement préhistorique de Duruthy à Sorde l'Abbaye (Landes). Bilan des recherches de 1958 à 1975. Paris, Mémoire de la Société préhistorique française, 1978. 158 p.

BAHN P.G. 1983 - Pyrenean Prehistory : a palaeoeconomic survey of the French sites.Warminster, Arris and Phillips Ltd., 1983. 511 p.

BANNIKOV A.G. 1958 - Distribution géographique actuelle et biologie de la Saïga en Europe. Mammalia, 1958, 22, p. 208-225.

BANNIKOV A.G. 1963 - Die Saiga-Antilope (Saiga tatarica L.). Wittenberg, Ziemsen, 1963. 143 p. 
BANNIKOV A.G. (dir.) 1967 - Biology of the Saiga. Jerusalem, Israel Program for Scientific Translation, 1967. $252 \mathrm{p}$.

BARTRAM L.E. 1993a - An Ethnoarchaeological Analysis of Kua San (Botswana) Bone Food Refuse. University of Wisconsin-Madison, 1993. $824 \mathrm{p}$.

BARTRAM L.E. 1993b - Perspectives on skeletal part profiles and utility curves from eastern Kalahari ethnoarchaeology. In : J. Hudson (Ed.), From Bones to Behavior : Ethnoarchaeological and Experimental Contributions to the Interpretation of Faunal Remains, p. 115-137. Carbondale, Center for Archaeological Investigations, Southern Illinois University at Carbondale.

BARTRAM L.E. et MAREAN C.W. 1999 - Explaining the pattern of bovid skeletal element abundance at Klasies River Mouth, South Africa : Kua ethnoarchaeology, the Die Kelders Middle Stone Age archaeofauna, long bone fragmentation, and carnivore ravaging. Journal of Archaeological Science, 1999, 26, p. 9-29.

BINFORD L.R. 1978 - Nunamiut Ethnoarchaeology. New York, Academic Press, 1978. 509 p.

BINFORD L.R. 1981 - Bones : Ancient Men and Modern Myths. New York, Academic Press, 1981. 320 p.

BLACK T.K. 1978 - A new method for assessing the sex of fragmentary skeletal remains femoral shaft circonference. American Journal of Physical Anthropology, 1978, 48, p. 227-231.

BLUMENSCHINE R.J. 1991 - Prey size and age models of prehistoric hominid scavenging : test cases from the Serengeti. In : M.C. Stiner (Ed.), Human predators and prey mortality, p. 122-147. Boulder, Westview press.

BOUCHUD J. 1951 - Etude paléontologique de la faune d'Isturitz. Mammalia, 1951, XV, p. 184-203.

BRAIN C.K. 1981 - The Hunters or the Hunted? An Introduction to African Cave Taphonomy. Chicago, University of Chicago Press, 1981. 365 p.

BROUGHTON J.M. 1994 - Late Holocene Resource Intensification in the Sacramento Valley, California : The Vertebrate Evidence. Journal of Archaeological Science, 1994, 21, p. 501-514.

BURKE A. 1995 - Prey movements and settlement patterns during the Upper Palaeolithic in Southwest France. Oxford, British Archaeological Reports (International Series), 1995. 126 p.

CASTEL J.-C. 1999 - Comportements de subsistance au Solutréen et au Badegoulien d'après les faunes de Combe-Saunière (Dordogne) et du Cuzoul de Vers (Lot). Thèse d'Université, Université Bordeaux I, 1999. $619 \mathrm{p}$.

CHEYNIER A. 1949 - Badegoule - station solutréenne et protomagdalénienne. Archives de l'Institut de Paléontologie humaine, Paris, 1949. 228 p.

CHEYNIER A., BOUCHUD J. et GUILLIEN Y. 1952 - Les bois de Renne de Badegoule. Bulletin de la Société Préhistorique Française, 1952, XLIX, p. 53-55.

COSTAMAGNO S. 1999 - Stratégies de chasse et fonction des sites au Magdalénien dans le sud de la France. Thèse d'Université, Université de Bordeaux I, 1999. 2 t., 495 p., 329 tabl., 132 fig., 128 pl.

COSTAMAGNO S. 2000 - Stratégies d'approvisionnement et traitement des carcasses au Magdalénien : l'exemple de Moulin-Neuf (Gironde). Paleo, 2000, 12, p. 77-95.

COSTAMAGNO S. soumis-a - Laboratory Taphonomy - Material loss and skeletal part profiles : The example of Saint-Germain-la-Rivière (Gironde, France). Archaeometry.

COSTAMAGNO S. soumis-b - Si les Magdaléniens du Sud de la France n'étaient pas des chasseurs spécialisés, qu'étaient-ils? Bulletin de la Société préhistorique française. 
COUSTÉ R. 1951 - Gisement magdalénien des grottes de Jaurias. Bulletin de la Société Préhistorique Française, 1951, 48, p. 381-384.

CRÉGUT-BONNOURE E. 1991 - L'environnement animal dans le midi de la France. Les dossiers d'Archéologie, 1991, 156, p. 48-63.

CRÉGUT-BONNOURE E. et GAGNIÈRE S. 1981 - Sur la présence de Saiga tatarica (MAMMALIA ARTIODACTYLA) dans le dépôt pléistocène supérieur de la grotte de la Salpêtrière à Remoulins (Gard, France). Nouvelles Archives du Museum d'Histoire naturelle de Lyon, 1981, 19, p. 37-42.

DAVID P. 1952 - Présence du Saïga dans le Moustérien ancien de la Chaise. Bulletin de la Société Préhistorique Française, 1952, 49, p. 68.

DAVID P. et PRAT F. 1965 - Considérations sur les faunes de la Chaise (commune de Vouthon, Charente) : Abri Suard et Bourgeois-Delaunay. Bulletin de l'association française pour l'étude du Quaternaire, 1965, 3-4, p. 222-231.

DELPECH F. 1971 - L'abri Faustin, commune de Cessac (Gironde). Etude paléontologique. Bulletin de la Société Préhistorique Française, 1971, 68, p. 328-332.

DELPECH F. 1972 - Fouilles de sauvetage dans le gisement magdalénien de Fongaban, commune de Saint-Emilion (Gironde) - $3^{\mathrm{eme}}$ partie : la Faune. L'Anthropologie, 1972, 76, p. 595-629.

DELPECH F. 1983 - Les faunes du Paléolithique supérieur dans le Sud-Ouest de la France. Paris, Cahier du Quaternaire, C.N.R.S., 1983. 453 p.

DELPECH F. 1987 - L'environnement animal des magdaléniens. In : M. Otte (Ed.), Le Magdalénien en Europe, p. 5-30. Actes du Colloque de Mayence, $\mathrm{XI}^{\mathrm{eme}}$ congrès U.I.S.P.P., ERAUL.

DELPECH F. 1989 - Le temps de l'Antilope saïga. In : J.-P. Mohen (Ed.), Le temps de la Préhistoire, p. 48-49. Paris, Sociéte préhistorique française et Archeologia.

DELPECH F. et HEINTZ E. 1976 - Les Artiodactyles : Bovidés. In : H.d. Lumley (Ed.), La Préhistoire Française. Tome 1. Les civilisations paléolithiques et mésolithiques de la France, p. 386-394. Paris, C.N.R.S.

DELPECH F. et LE GALL O. 1983 - La faune magdalénienne de la grotte des Eglises (Ussat, Ariège). Bulletin de la Société Préhistorique de l'Ariège, 1983, 36, p. 91-118.

DELPECH F. et PRAT F. 1995 - Nouvelles observations sur les faunes acheuléennnes de CombeGrenal (Domme, Dordogne),. Paleo, 1995, 7, p. 123-137.

DELLUC B. et DELLUC G. 1981 - La grotte ornée de Comarque à Sireuil (Dordogne). Gallia Préhistoire, 1981, 24, p. 1-97.

DENIZ E. et PAYNE S. 1982 - Eruption and wear in the mandibular dentition as a guide to ageing turkish Angora Goats. In : B. Wilson, C. Grigson et S. Payne (Eds.), Aging and sexing animal bones from archaeological sites, p. 155-205. Oxford, British Archaeological Reports (International Series).

DEPLANO S. 1994 - Etude de la faune des grands mammifères de la couche IX de l'abri du Flageolet II Dordogne. Approche taphonomique et palethnographique. Mémoire de Maîtrise, Université de Paris X, 1994. $88 \mathrm{p}$.

DETRAIN L., GUILLON M., KERVAZO B., MADELAINE S., MORALA A. et TURQ A. 1996 - Le Moulin du Roc à Saint-Chamassy (Dordogne) - Résultats préliminaires. Bulletin de la Société Préhistorique Française, 1996, 93, p. 43-48.

DRIVER J.C. 1990 - Meat in due season : the timing of communal hunts. In : L.B. Davis et B.O.K. Reeves (Eds.), Hunters of the Recent Past, p. 11-33. London, Unwin Hyman. 
FARIZY C., DAVID F. et JAUBERT J. (dir.) 1994 - Hommes et bisons du Paléolithique moyen à Mauran (Haute-Garonne). Paris, C.N.R.S, 1994. 259 p.

FISCHER D.C. 1987 - Mastodont procurement by paleoindians of the Great Lakes Region : hunting or scavenging. In : M.H. Nitecki et D.V. Nitecki (Eds.), The Evolution of Human Hunting, p. 309-421. New-York, Plenum Press.

FONTANA L. 1998a - Mobilité et subsistance au Magdalénien supérieur et final en Auvergne. In : J.-P. Brugal, L. Meignen et M. Patou-Mathis (Eds.), Economie préhistorique : les comportements de subsistance au Paléolithique, p. 373-386. XVIII ${ }^{\text {eme }}$ Rencontres Internationales d'Archéologie et d'Histoire d'Antibes, APCDA-CNRS.

FONTANA L. 1998b - Subsistance et territoire au Magdalénien supérieur dans les Pyrénées : l'apport des données archéozoologiques de la grotte de Belvis. Bulletin Préhistore du sud-Ouest, Nouvelles Etudes, 1998, 5, p. 131-146.

FONTANA L. 1999 - Mobilité et subsistance au Magdalénien dans le Bassin de l'Aude. Bulletin de la Société Préhistorique Française, 1999, 96, p. 175-190.

FRISON G.C. 1991 - Hunting strategies, prey behavior and mortality data. In : M.C. Stiner (Ed.), Human predators and prey mortality, p. 15-30. Boulder, Westview press.

GAUSSEN J. 1959 - Un gisement magdalénien dans la vallée de l'Isle. L'abri Jumeau, à Soursac (Dorgogne). Bulletin de la Société Préhistorique Française, 1959, 56, p. 487-492.

GILBERT A. 1984 - Contribution à l'étude des faunes de la fin des temps glacaires et du début des temps postglacaires. Thèse de troisième cycle, Université de Bordeaux I, 1984. 322 p., 132 tabl., 10 fig., 9 pl.

GORDON B. 1988 - Of men and reindeer herds in french Magdalenian Prehistory. Oxford, British Archaeological Reports (International Series), 1988. 233 p.

GRIGGO C. 1995 - Significations paléoenvironnementales des communautés animales pléistocènes reconnues dans l'abri Suard (Charente) et la Grotte de Bois-Ragot (Vienne) : Essai de quantification de variables climatiques. Thèse d'Université, Université de Bordeaux I, 1995. 206 p.

GRIGSON C. 1987 - Different herding strategies for sheep and goats in the Chalcolithic of Beersheva. Archaeozoologia, 1987, 1, p. 115-126.

GUADELLI J.-L. 1987 - Les zoocénoses préhistoriques en Aquitaine (Würm ancien et interstade würmien). Thèse d'Université, Université de Bordeaux I, 1987. 3 t., 568 p., 424 tabl.,163 fig.

GUADELLI J.-L. et OZOUF J.-C. 1994 - Etudes expérimentales de l'action du gel sur les restes fauniques : Premiers résultats. In : M. Patou-Mathis (Ed.), Outillage peu élaboré en os et bois de Cervidés, 6eme Table Ronde, Taphonomie Bone Modification, p. 47-56. Paris, CEDARC.

KOIKE H. et OHTAISHI N. 1985 - Prehistoric hunting pressure estimated by the age composition of excavated Sika deer (Cervus nippon) using the annual layer of tooth cement. Journal of Archaeological Science, 1985, 12, p. 443-456.

KOIKE H. et OHTAISHI N. 1987 - Estimation of prehistoric hunting rates based on the age composition of Sika Deer (Cervus nippon). Journal of Archaeological Science, 1987, 14, p. 251-269.

LARSON J.S. et TABER R.D. 1980 - Criteria of sex and age, Wildlife Management Technique Manual, p. 143-202. The Wildlife Society. LARSON J.S. et TABER R.D. 1980 - Criteria of sex and age, Wildlife Management Technique Manual, p. 143-202. The Wildlife Society.

LENOIR M. 1983 - Le Paléolithique des Basses Vallées de la Dordogne. Thèse d'Etat, Université de Bordeaux I, 1983. 3 t., 702 p., 462 fig., 41 tabl. 
LENOIR M. 1996 - Hommes et matières premières au Pléistocène en Gironde. Bulletin Préhistoire du sud-Ouest, Nouvelles études, 1996, 3, p. 147-151.

LENOIR M., OBRY J. et SERONIE-VIVIEN M.-R. 1997 - Occurence of allochtonous flint in an UpperPaleolithic site near Bordeaux. In : A. Ramos-Millan et M.A. Bustillo (Eds.), Siliceous Rock and Culture, p. 385-390. Granada, Universidad de Granada.

LE TENSORER J.-M. 1973 - Le gisement du Martinet à Sauveterre-la-Lemance (Lot-et-Garonne) Etude géologique et géochimique. Bulletin de l'Association française pour l'étude du Quaternaire, 1973, 36, p. 215-237.

LE TENSORER J.-M. 1981 - Le Paléolithique de l'Agenais. Paris, Cahier du Quaternaire, C.N.R.S., 1981. $523 \mathrm{p}$.

LOWE V. 1967 - Teeth as indicators of age with special reference to red deer (Cervus elaphus). Journal of Zoology (London), 1967, 152, p. 137-153.

LYMAN R.L. 1984 - Bone density and differential survivorship of fossil classes. Journal of Anthropological Archaeology, 1984, 3, p. 259-299.

LYMAN R.L. 1991 - Subsistence change and pinniped hunting. In : M.C. Stiner (Ed.), Human predators and prey mortality, p. 187-199. Boulder, Westview press.

MAGNY M. 1995 - Une histoire du climat - Des derniers mammouths au siècle de l'automobile. Paris, Editions Errance, 1995. 176 p.

MAREAN C.W. 1998 - A critique of the evidence for scavenging by neandertals and early modern humans : new data from Kobeh Cave (Zagros Mountains, Iran) and Die Kelders Cave 1 Layer 10 (South Africa). Journal of Human Evolution, 1998, 35, p. 111-136.

MAREAN C.W., BLUMENSCHINE R.J., SPENCER L.M. et CAPALDO S. 1992 - Captive hyaena bone choice and destruction, the schlepp effect, and Olduvai archaeofaunas. Journal of Archaeological Science, 1992, 19, p. 101-121.

MAREAN C.W. et FREY J. 1997 - The animal bones from caves to cities : Reverse utility curves as methodological artifacts. American Antiquity, 1997, 62, p. 698-711.

MARIEZKURRENA K. et ALTUNA J. 1983 - Biometria y diformismo sexual en el esqueleto de Cervus elphus würmiense, postwürmiense y actual del Cantabrico. Munibe, 1983, 35, p. 203-246.

NOBLE W.C. et CRERAR J.E.M. 1993 - Management of white-tailed deer by the neutral iroquois A.D. 999-1651. Archaeozoologia, 1993, 6, p. 19-70.

O'CONNELL J.F., HAWKES K. et BLURTON-JONES N. 1990 - Reanalysis of large mammal body part transport among the Hadza. Journal of Archaeological Science, 1990, 17, p. 301-316.

OUCHAOU B. 1985 - La faune du gisement de la Lustre. Mémoire de D.E.A., Université de Bordeaux I, 1985. $71 \mathrm{p}$.

OUZRIT L. 1986 - Recherches sur les faunes du Dryas ancien en Gironde. Le gisement de Saint-Germain-laRivière. Thèse de 3eme cycle, Université de Bordeaux I, 1986. 147 p., 158 tabl., 44 fig.

PAILHAUGUE N. 1998 - Faune et saisons d'occupation de la salle Monique au Magdalénien pyrénéen, Grotte de la Vache (Alliat, Ariège, France). Quaternaire, 1998, 9, p. 385-400.

PAYNE S. 1973 - Kill-off patterns in sheep and goats : the mandibles from Asvan Kale. Anatolian Studies, 1973, 23, p. 282-303.

PERKINS D. et DALY P. 1968 - A hunter's village in Neolithic Turkey. Scientific American, 1968, 219, p. 97-106. 
PFEFFER P. 1964 - Le rôle des facteurs climatiques dans la dynamique des populations d'Ongulés sauvages des steppes et déserts paléarctiques. La terre et la vie, 1964, 111, p. 167-177.

PURDUE J.R. 1983 - Methods of determining sex and body size in prehistoric samples of whitetailed deer (Odocoileus virginianus). Transactions of the Illinois State Academy of Science, 1983, 76, p. 351-357.

ROUSSOT A. 1962 - Le gisement paléolithique de Reignac, commune de Tursac. Bulletin de la Société Historique et Archéologique du Périgord, 1962, 89, p. 145-146.

ROUSSOT A. 1964 - Le gisement paléolithique de Reignac, commune de Tursac. Bulletin de la Société Historique et Archéologique du Périgord, 1964, 91, p. 63-70.

ROUSSOT A. 1978 - La Maison-Forte-de-Reignac. Gallia Préhistoire, 1978, 21, p. 633-635.

SAINT-PERIER R., (de) 1936 - La grotte d'Isturitz - Le Magdalénien de la Salle de Saint-Martin. Paris, Archives de l'Institut de Paléontologie humaine, Masson, 1936. 120 p.

SIMMS S.R. 1987 - Behavioral ecology and hunter-gatherer foraging - An example from the Great Basin. Oxford, British Archaeological Reports (International Series), 1987. 157 p.

SLOTT-MOLLER R. 1988 - Contribution à l'étude paléontologique d'un gisement préhistorique : l'exemple du Roc de Marcamps (Gironde). Diplôme d'étude supérieures de Sciences Naturelles. Université de Bordeaux I, 1988. 173 p., 146 tabl., 70 fig.

SPETH J.D. 1983 - Bison Kills and Bone Counts : Decision Making by Ancient Hunters. Chicago, University of Chicago Press, 1983. 227 p.

SPETH J.D. 1991 - Taphonomy and early hominid behavior : problems in distinguishing cultural and non-cultural agents. In : M.C. Stiner (Ed.), Human predators and prey mortality, p. 31-40. Boulder, Westview press.

STEEL F.L.D. 1962 - The sexing of long bones, with reference to the St. Bride's series of identified skeletons. Royal Anthropological Institute of Great Britain and Ireland, 1962, 92, p. 212-222.

STINER M.C. 1990 - The use of mortality patterns in archaeological studies of hominid predatory adaptations. Journal of Anthropological Archaeology, 1990, 9, p. 305-351.

STINER M.C. (dir.) 1991a - Human predators and prey mortality. Boulder, Westview Press, 1991. $276 \mathrm{p}$.

STINER M.C. 1991b - An interspecific perspective on the emergence of the modern human predatory niche. In : M.C. stiner (Ed.), Human predators and prey mortality, p. 149-185. Boulder, Westview press.

STINER M.C. 1994 - Honor Among Thieves. Princeton, N.J., Princeton University Press, 1994. 447 p.

STRAUS L.G. 1992 - L'abri Dufaure et la falaise du Pastou dans le système adaptatif régional des Pyrénées au Magdalénien, Le Peuplement magdalénien. Paléogéographie physique et humaine, $\mathrm{p}$. 335-344. Actes du colloque de Chancelade, C.T.H.S.

STRAUS L.G. (dir.) 1995 - Les derniers chasseurs de rennes du monde pyrénéen. L'abri Dufaure : un gisement tardiglaciaire en Gascogne. Paris, Mémoire de la Société Préhistorique française, 1995. $287 \mathrm{p}$.

TEXIER J.-P., BERTRAN P., COUTARD J.-P., FRANCOU B., GABERT P., GUADELLI J.-L., OZOUF J.-C., PLISSON H., RAYNAL J.-P. et VIVENT D. 1998 - TRANSIT, an experimental archaeological program in periglacial environment : problematic, methodology, first results. Geoarchaeology, 1998, 13, p. $1-41$. 
THOMAS D.H. et MAYER D. 1983 - Behavioral faunal analysis of selected horizons. In : D.H.

Thomas (Ed.), The Archaeology of Monitor Valley : 2. Gatecliff Shelter, p. 353-391. New York, American Museum of Natural History.

TODD L.C. 1986 - Determination of sex of Bison upper forelimb bones ; the humerus and radius. The Wyoming Archaeologist, 1986, 29, p. 103-124.

TOURNEPICHE J.-F. 1982 - Le gisement paléontologique würmien de la grotte du Quéroy (Charente). Bulletin de la Société Préhistorique Française, 1982, 79, p. 99.

TOURNEPICHE J.-F. 1996 - Les grands mammifères pléistocènes de Poitou-Charente. Paleo, 1996, 8, p. 109-141.

WHEAT J.B. 1972 - The Olsen Chubbuck Site : A Paleo Indian Bison Kill. American Antiquity, 1972, 37, p. 1-181.

\section{NOTES}

1. Les niveaux où ont été découverts les squelettes d'Antilope saïga du Quéroy sont datés de 12100 BP. Or d'après F. Delpech, 1987, à la fin du stade 2, l'aire de répartition de l'Antilope Saïga diminue fortement ; elle quitte les plaines d'Aquitaine. La Charente pourrait donc représenter, comme le Sud-Est, une des zones refuges de l'Antilope avant sa complète disparition de France. Cette diminution de l'aire géographique de la Saïga serait le résultat de la mise en place de conditions climatiques beaucoup moins favorables au développement de cette espèce. Au cours du Würm récent, la taille du Renne varie en fonction des conditions climatiques (Delpech, 1983 ; Griggo, 1995). La faible taille des individus récoltés au Quéroy par rapport à ceux de SaintGermain-la-Rivière pourrait résulter du même phénomène.

2. Pour la discussion, seules les couches 1 et 3 sont prises en compte, les résultats des couches $C$ et 4 étant respectivement comparables à ceux des niveaux 1 et 3 (Costamagno 1999).

3. L'inverse de l'indice de Simpson permet d'évaluer la diversité des ensembles osseux $(1 / \Sigma$ pi2 où pi $=$ NISPi/NISP et $\mathrm{i}$ est un taxon donné) ; plus la valeur est forte, plus le spectre faunique est diversifié.

\section{RÉSUMÉS}

L'Antilope saïga a connu deux phases de migration : l'une durant le stade isotopique 6, l'autre durant le stade isotopique 2. Cette seconde vague de migration beaucoup mieux documentée que la première a eu un fort impact en Aquitaine. En effet, c'est la seule zone où l'Antilope saïga a joué un rôle non négligeable dans l'alimentation des Magdaléniens. Afin d'évaluer l'influence de cette espèce sur les stratégies de chasse et les modalités d'exploitation des territoires, un chapitre est consacré à l'écologie et à l'éthologie de cet animal. Une méthode d'estimation d'âge des antilopes saïga à partir des restes dentaires est ensuite présentée ainsi qu'une étude sur les os post-crâniens permettant une discrimination des mâles et des femelles.

L'étude montre que l'exploitation de l'Antilope saïga en Aquitaine est restreinte dans le temps (dernier Pléniglaciaire et Dryas ancien) et dans l'espace (Gironde). En Gironde, l'analyse archéozoologique détaillée de Saint-Germain-la-Rivière et de Moulin-Neuf montre que les 
antilopes saïga étaient présentes dans cette zone saisonnièrement. Les hommes pratiquaient alors une chasse opportuniste à petite échelle sur les hardes mixtes présentes dans l'environnement. Le reste de l'année, ils continuaient à occuper la région en exploitant d'autres espèces (Cheval, Renne, Bovinés).

For the Saiga antelope, two major phases of migration have been recognized : the first one during oxygen isotope 6 , the other one during oxygen isotope 2 . This second wave is better documented in Aquitaine than the first one. Indeed, it is the only region where the Saiga antelope plays a large role in Magdalenian subsistence. To evaluate the influence of the Saiga antelope on hunting strategies and the mobility of Magdalenian people, the first chapter is dedicated to the ecology of the Saiga antelope. Then, a technique for the determination of Saiga antelope mandibular teeth is presented, as well as a study of post-cranial bones to discriminate between males and females. The studies show that the Saiga antelope's exploitation in Aquitaine was restricted in time and in space. In Gironde, the zooarchaeological analyses of Saint-Germain-la-Rivière and Moulin-Neuf suggest that the Saiga antilope was present in this region only seasonally. Then, a Saiga hunting in small scale occurred at random within the mixed herd. During the rest of the year, human groups continued to occupy the region by exploiting other taxa (horse, reindeer, large bovids). It appears that the Gironde environment supplied hunter-gatherers with resources year-long.

\section{INDEX}

Keywords : ecology, Magdalenian, Saiga antelope, seasonality, subsistence strategies, technique for age-scoring, territory, zooarchaeology

Mots-clés : Antilope saïga, archéozoologie, économie de subsistance, éthologie, Magdalénien, méthode d'estimation d'âge, saisonnalité, territoire

\section{AUTEUR}

\section{SANDRINE COSTAMAGNO}

Maison de la Recherche - U.M.R. 5608, Université de Toulouse 2 - Le Mirail, 5 allées Antonio Machado, 31058 Toulouse cedex 